\title{
A New Design Method for Optimal Parameters Setting of PSSs and SVC Damping Controllers to Alleviate Power System Stability Problem
}

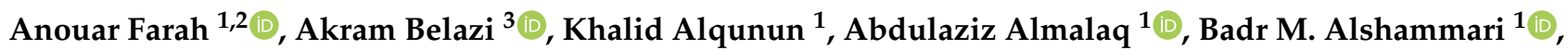 \\ Mohamed Bechir Ben Hamida ${ }^{4,5, * \mathbb{C}}$ and Rabeh Abbassi ${ }^{1}$
}

check for

updates

Citation: Farah, A.; Belazi, A.

Alqunun, K.; Almalaq, A.;

Alshammari, B.M.; Ben Hamida, M.B.;

Abassi, R. A New Design Method for

Optimal Parameters Setting of PSSs

and SVC Damping Controllers to

Alleviate Power System Stability

Problem. Energies 2021, 14, 7312.

https://doi.org/10.3390/en14217312

Academic Editors: Reinaldo Tonkoski and James Cale

Received: 17 September 2021

Accepted: 30 October 2021

Published: 4 November 2021

Publisher's Note: MDPI stays neutral with regard to jurisdictional claims in published maps and institutional affiliations.

Copyright: (c) 2021 by the authors Licensee MDPI, Basel, Switzerland. This article is an open access article distributed under the terms and conditions of the Creative Commons Attribution (CC BY) license (https:/ / creativecommons.org/licenses/by/ $4.0 /)$
1 Department of Electrical Engineering, College of Engineering, University of Ha'il, Ha'il 2240, Saudi Arabia; farah.anouar@gmail.com (A.F.); kh.alqunun@uoh.edu.sa (K.A.); a.almalaq@hotmail.com (A.A.); bms.alshammari@uoh.edu.sa (B.M.A.); r_abbassi@yahoo.fr (R.A.)

2 Department of Electrical Engineering, National Engineering School of Sfax, University of Sfax, Sfax 3038, Tunisia

3 Laboratory RISC-ENIT (LR-16-ES07), Tunis El Manar University, Tunis 1002, Tunisia; akram.belazi@enit.utm.tn

4 Department of Chemical Engineering, College of Engineering, University of Ha'il, Ha'il 2240, Saudi Arabia

5 Research Laboratory of Ionized Backgrounds and Reagents Studies, Preparatory Institute for Engineering Studies of Monastir, University of Monastir, Monastir 5019, Tunisia

* Correspondence: benhamida_mbechir@yahoo.fr

\begin{abstract}
This paper presents an improved Teaching-Learning-Based Optimization (TLBO) for optimal tuning of power system stabilizers (PSSs) and static VAR compensator (SVC)-based controllers. The original TLBO is characterized by easy implementation and is mainly free of control parameters. Unfortunately, TLBO may suffer from population diversity losses in some cases, leading to local optimum and premature convergence. In this study, three approaches are considered for improving the original TLBO (i) randomness improvement, (ii) three new mutation strategies (iii) hyperchaotic perturbation strategy. In the first approach, all random numbers in the original TLBO are substituted by the hyperchaotic map sequence to boost exploration capability. In the second approach, three mutations are carried out to explore a new promising search space. The obtained solution is further improved in the third strategy by implementing a new perturbation equation. The proposed HTLBO was evaluated with 26 test functions. The obtained results show that HTLBO outperforms the TBLO algorithm and some state-of-the-art algorithms in robustness and accuracy in almost all experiments. Moreover, the efficacy of the proposed HTLBO is justified by involving it in the power system stability problem. The results consist of the Integral of Absolute Error (ITAE) and eigenvalue analysis of electromechanical modes demonstrate the superiority and the potential of the proposed HTLBO based PSSs and SVC controllers over a wide range of operating conditions. Besides, the advantage of the proposed coordination design controllers was confirmed by comparing them to PSSs and SVC tuned individually.
\end{abstract}

Keywords: power system stability; electromechanical oscillations; PSS; SVC; TLBO; hyperchaotic TLBO

\section{Introduction}

\subsection{Research Background}

In several engineering problems, acceptable solutions are generally not reached using conventional algorithms at a reasonable cost and time. Generally, the problems as mentioned earlier are multimodal. Recently, several methods have been involved in the resolution of engineering problems. These techniques are characterized by different approaches in the process of finding the optimal solutions. Some of these methods imitate the natural processes and are named metaheuristics. Actually, the latter techniques have shown concurrent results for handling harsh engineering problems such as power system dispatch 
and stability [1,2], energy commitment problems [3], energy management of micro-grid [4], and manufacturing industry [5].

\subsection{Literature Review}

The electromechanical oscillations are observed in the power system following the unbalance between mechanical and electrical torques at the synchronous generator, caused by the variation of power system topology or loads. When these oscillations are poorly damped, the generator rotor shaft and the power transfers are highly affected. Generally, the electromechanical oscillations can be classified into two types: the inter-area and local modes, which range between 0.1 and $1 \mathrm{~Hz}$ and $1-2 \mathrm{~Hz}$, respectively [6]. The local modes are recognized as the oscillation of the generation units at one station with respect to the rest of the power system. The inter-area mode referred to the oscillation of many generation units in a given area against machines in other parts [7]. The reliability and security of a power system are highly affected by these oscillations.

Power system operators routinely use the Power System Stabilizer (PSS) to alleviate the electromechanical oscillations of synchronous machines. Unfortunately, some weakness is encountered in the damping of inter-area oscillations, and other solutions need to be involved. FACTS controllers, which are based on power electronics, give more flexibility to the existing power system [8]. In other words, they can increase power transfer capability, improve power system stability and controllability. Static Var Compensator (SVC) is the most installed type of FACTS. Furthermore, other proprieties of the power system can be improved, such as dynamic control of power flow, steady-state stability limits, and damping of electromechanical oscillations [9]. SVC acts by introducing to the transmission network a variable reactive admittance. Both kinds of oscillations are enhanced when an SVC device is used in conjunction with PSSs. Considering the limitation of other PSSs structure and the easiness of implementation, lead-lag PSSs is chosen by the power systems utilities. The efficacy of PSSs is highly related to the fine selection of its parameters. Conventional approaches generally fail to solve the problem design of PSSs [10].

The problem design of power system controllers parameters is formulated as a nondifferentiable and large-scale nonlinear problem. This optimization problem is harsh to solve by employing traditional optimization techniques such as sequential quadratic programming (SQP) techniques due to their high sensitivity to the initial point. Furthermore, these methods require a long time in the convergence process. To overcome the drawbacks mentioned earlier, intelligent techniques are involved in real-life engineering problems, including power system stability. Intelligent techniques can be classified into two types: evolutionary and swarm algorithms. Evolutionary algorithms are inspired by the natural evolution process. In this class, the most popular algorithm is the Genetic Algorithm (GA) [11], which is based on the Darwinian theory of the evolution process. Unfortunately, researchers revealed some deficiencies in GA, especially when the parameters to be optimized are highly correlated. Among evolutionary algorithms that have been successfully applied in different areas, we cite: Differential Evolution (DE) [12], Genetic programming [13], Evolution strategy and Biogeography-Based Optimization (BBO) algorithm [14]. The swarm techniques imitate the collective behavior of groups of animals. The most known swarm optimization algorithm is the Particle Swarm Optimization inspired by the behavior of bird flocking. PSO was first introduced by Kennedy and Eberhart [15]. Another famous swarm technique is Ant Colony Optimization (ACO) [16] inspired by the behavior of ants for tracking the shortest path between the source food and the nest. Several swarm techniques are applied in various domains such as Cuckoo Search (CS) algorithm [17], Artificial Bee Colony (ABC) algorithm [18], Firefly Algorithm (FA) [19], Bat Algorithm (BA) [20] and Harmony Search (HS) [21].

Recently, intelligent techniques that mimic human behavior have emerged. Some of algorithms belonging to this class are: Imperialist Competitive Algorithm (ICA) [22], Firework Algorithm [23], Seeker Optimization Algorithm (SOA) [24] and Interior Search Algorithm (ISA) [25]. TLBO [26] is the most popular algorithm inspired by human behavior. 
TLBO Simulates the teaching-learning process in a class. It is based on the influence of the teacher on their learners. Furthermore, by an interaction between themselves, the learners can achieve a positive contribution to enhance grades. The strong points of the TLBO algorithm are easy implementation, small required computational memory, free of control parameters in all equations that simulate the updating process of output knowledge of learners. Several researchers have successfully applied the TLBO in real-engineering problems in various fields. Some weakness is encountered in standard TLBO, especially when the problems to be optimized revealed an enormous number of local optima. Recently, several improvements to TLBO have been suggested by researchers. In $[27,28]$, the authors modify the learning process by using a new mutation strategy to enhance the diversity of solutions. Then, the improved TLBO was employed to solve non-smooth optimal power flow. In [29], an elitist TLBO algorithm is introduced to solve the petrochemical industry planning problem. The dynamic group strategy concept, which consists of improving the output of a given learner from the mean of their group, was involved in improving the global search capacity. Bi-phase crossover and local search strategy are used to reach a perfect balance between exploration and exploitation capabilities in [30]. The updating equations of TLBO, which simulate the teaching and learning process, are modified using a scale factor [31]. A modified mutation operator and a new teacher factor alleviate some drawbacks of the standard TLBO by avoiding premature convergence on a local optimum. In [32], a group discussion is introduced as a powerful method to increase the efficiency of standard TLBO by involving a group of leaders. Another approach to enhance the abilities of the standard TLBO is to boost the learning phase by adding a self-learning phase [33]. A new hybrid optimization algorithm is developed by combining the TLBO algorithm and the Artificial bee colony algorithm (ABC) for prediction of the berm geometry [34]. A multi-objective optimization algorithm developed by integrating the DE in TLBO obtains the optimal solution of a hydro-thermal scheduling problem. Multi-objective TLBO is introduced in [35] to overcome the problem of optimal power flow. The learning knowledge of other students is integrated into TLBO to boost learners' learning speed and output knowledge. The obtained algorithm is tested on the design problems of the PID controller and a gear train in addition to 18 test functions.

Several intelligent techniques have been suggested for designing SVC to damp out electromechanical oscillations and enhance power system dynamic stability. A bee colony (ABC) algorithm with the sequential quadratic programming (SQP) optimization technique was employed for the tuning of PSS and SVC-based controllers in [36]. The implementation of SVC using wavelet neural network to improve power system stability is presented in [37]. A method for seeking the best-input signals and the optimal location of several SVC involving a stability index was developed in [38]. The optimal setting of SVC for voltage stability enhancement was addressed in [39]. In [40] a coordination tuning of PSS and SVC-based controller employing bacterial swarming optimization (BSO) has been performed to achieve optimal damping of electromechanical oscillations. The design of the SVC parameters using a shuffled frog-leaping algorithm to enhance power system stability was presented in [41]. In [42], the authors have suggested an optimal allocation, size, and parameters of the SVC using multi-objective PSO. In [43], the researchers have been involved the bacterial foraging optimization algorithm (BFOA) for achieving the coordination of SVC and PSS to attain optimal damping performance of power system.

Recently, many researchers have embedded a new mathematical approach named chaos to replace algorithm-parameters in several optimization algorithms such as ABC algorithm [44], Chaotic crow search optimization algorithm [45], PSO [46], Jaya [47-50], Gravitational Search Algorithm (GSA) [51], and TLBO [2]. The chaos approach has nonrepetition and ergodicity properties, which increase the search behavior's speed and boost the generated solutions' diversity. Besides, chaos allows the balance between exploitation and exploration capabilities. The final solution provided by a given optimization algorithm can be improved by embedding the chaotic maps [44]. The solution quality of the original crow search optimization algorithm is enhanced by substituting the random numbers 
by chaotic scheme [45]. The search behavior and the convergence characteristic of the standard PSO are improved by using chaotic dynamic weight. All the aforementioned works demonstrate the benefit of a chaotic scheme compared to random numbers.

\subsection{Contributions}

The contributions of this work can be summarized as follows:

- The original TLBO is improved by considering randomness improvement, new mutation equations, and hyperchaotic perturbation scheme.

- The HTLBO is proposed for solving the power system stability problem.

- The performance of the HTLBO technique is proved by using 26 test functions with different comparative methods such as FA, PSO, DE, and TLBO.

- The statistical signification of the obtained results is achieved by involving the Friedman ranking scheme.

\subsection{Structure of the Manuscript}

The remainder of this work is introduced as follows: The standard TLBO and the 5D hyperchaotic map are detailed in Section 2. Section 3 present the proposed hyperchaotic teaching-learning algorithm. The numerical results were stated in Section 4 . The application of our approach to solving the power system stability design problem was discussed in Section 5. The conclusions were drawn in Section 6.

\section{Preliminaries}

In this part, the fundamental concepts of Teaching-Learning-Based Optimization are introduced. Furthermore, the basic knowledge of the hyperchaotic 5-D map is defined as well.

\subsection{TLBO}

TLBO is a population-based optimization that imitates the teaching and learning process. It was devised by Rao et al. and can be summarized in the teacher and learner phases. In the first phase, the teacher transmits their expertise to all students, whereas in the learner phase, students interact with their fellow to improve their knowledge. Under TLBO, the population is simulated by a class or a group of learners. In optimization algorithms, a design variable is associated with each individual in the population. Further details of TLBO can be found in [26,52]. More information on the TLBO phases is provided next part. Theoretically, in the teacher phase, a good teacher aims to transfer all their knowledge to each learner in the class. However, in practice, it cannot be reached due to the limited student's capabilities, and as a consequence, only the mean knowledge of the class can be improved. The $i$ th learners with d-dimensional variables can be defined as: $S_{i}=\left[s_{i 1}, s_{i 2}, \ldots, s_{i d}\right]$. The average of students grades can be represented by : Smean $=$ $\frac{1}{N L}\left[\sum_{i=1}^{N L} s_{i 1}, \sum_{i=1}^{N L} s_{i 2}, \ldots, \sum_{i=1}^{N L} s_{i d}\right]$ where NL is the number of students. It is noteworthy that the minimum fitness criterion was adopted to choose the teacher among all learners. The teaching process can be described as follows:

$$
S_{\text {new }}=S_{i}+\operatorname{rand}\left(S_{\text {teacher }}-\alpha S_{\text {mean }}\right),
$$

where rand is a random number, $\alpha$ is the teaching factor given by the following expression $: \alpha=\operatorname{round}(1+$ rand $)$. If the objective function of the new solution is lesser than the old one, it will be accepted. Contrarily, the new solution will be rejected.

Learners improve their knowledge through the teacher as well as by the interaction between themselves. The interaction process is performed by randomly selecting two different learners $S_{j}$ and $S_{k}$. The new learners is accepted in the population if he/she has the lesser objective function (2) and (3). If the previous condition is not satisfied, the population of students is kept unchanged. The new population of students is updated as follows: 
If $\operatorname{Obj}\left(S_{j}\right)<\operatorname{Obj}\left(S_{k}\right)$

$$
S_{j, \text { new }}=S_{j, o l d}+\operatorname{rand}_{j}\left(S_{j}-S_{k}\right)
$$

Else

$$
S_{j, \text { new }}=S_{j, \text { old }}+\operatorname{rand}_{j}\left(S_{k}-S_{j}\right)
$$

\subsection{The 5D Hyperchaotic System}

In [53], a new five-dimensional (5D) hyperchaotic system is presented. It is derived from the 3D-modified generalized Lorenz system, and it can display complex dynamical behaviors such as period, chaos, and hyperchaos. The dynamics of such a 5D can be mathematically expressed as:

$$
\left\{\begin{array}{l}
\dot{x}=a(y-x), \\
\dot{y}=c x+d y-x z+w, \\
\dot{z}=-b z+x^{2}, \\
\dot{u}=e y+f u \\
\dot{w}=-r x-k w,
\end{array}\right.
$$

where $a>0, b>0, d>-c, e r \neq 0, a, b, c, d$, and $f$ are constant parameters. $e$ and $w$ are the coupling parameter and the controller, respectively. $r$ and $k$ are the control parameters responsible for the periodic, chaotic, and hyperchaotic behaviors and bifurcations of the system. The hyperchaotic behavior of (4) is shown in Figures 1 and 2, which illustrate the 2D and 3D phase portraits of the hyperchaotic attractor ( $c f .(4))$, respectively.

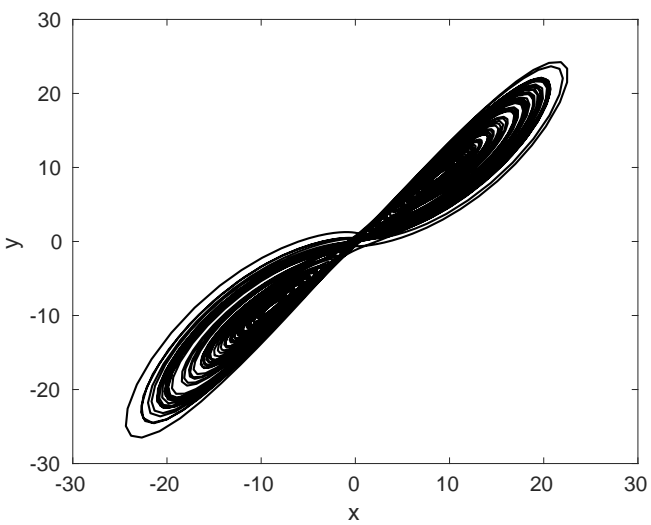

(a)

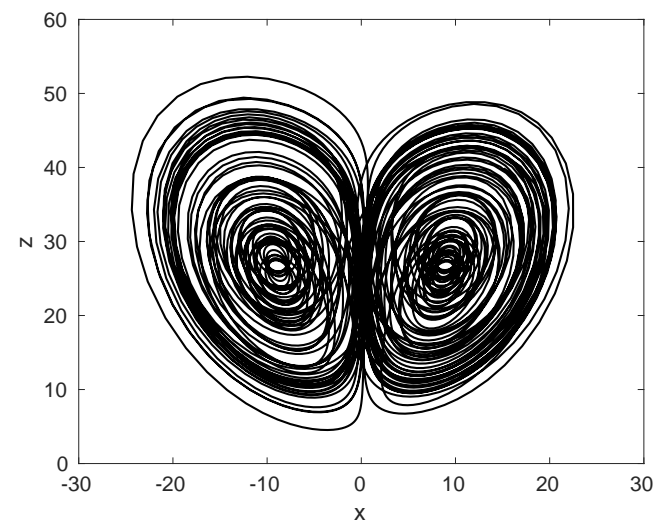

(b)

Figure 1. 2D phase portraits of the hyperchaotic attractor of (4) in $x-y$ plane (a), and in $x-z$ plane (b), where $(a, b, c, d, e, f, r, k)=(35,3,35,-8.1,1,1,1,0.05)$.

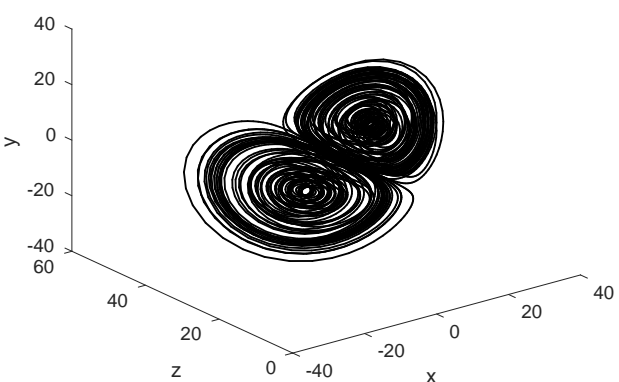

(a)

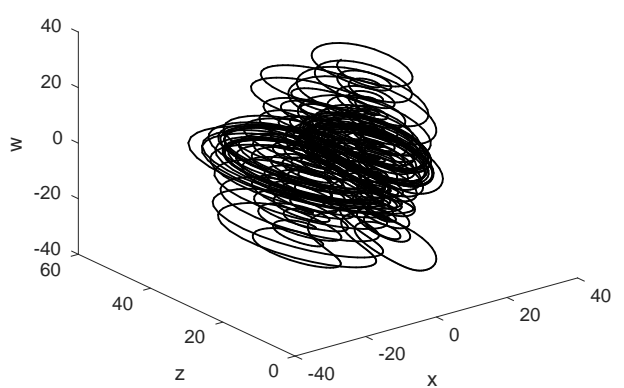

(b)

Figure 2. 3D phase portraits of the hyperchaotic attractor of (4) in $x-z-y$ space (a), and in $x-z-w$ space $(\mathbf{b})$, where $(a, b, c, d, e, f, r, k)=(35,3,35,-8.1,1,1,1,0.05)$. 


\section{Proposed Hyperchaotic Teaching-Learning-Based Optimization}

The improvements introduced in the hyperchaotic Teaching-Learning-Based Optimization are the randomness phase, three new search equations, and a hyperchaotic perturbation strategy.

\subsection{Randomness Improvement}

The procedure for finding the global optimum solution depends on randomness to imitate the teaching-learning process. Furthermore, the convergence speed is highly affected by random parameters. Consequently, it is beneficial to adopt non-conventional distributions (i.e., the hyperchaotic map) instead of the standard distributions (i.e., Gauss or uniform distributions).

The initial equation is described as follows:

$$
S_{j}^{i}=S_{\text {min }}^{i}+H c_{j}\left(S_{\text {max }}^{i}-S_{\text {min }}^{i}\right)
$$

Teacher phase: The teaching-learning process depends on the learner's influence and the quality of the teacher. In standard TLBO, the difference between the teacher and the class grades mean is involved in updating the level of learners. In the HTLBO, hyperchaotic numbers are used to replace the random sequence. The obtained hyperchaotic mutation can increase the level of learners. Furthermore, the newly added term $H_{c, i} \cdot\left(X_{\text {worst }}-\left|X_{o l d, i j}\right|\right)$ express the tendency to avoid the worst solution. The implementation can be encapsulated as follows:

$$
S_{\text {new }, i j}=S_{o l d, i j}+H_{c, i}\left(S_{\text {Teacher }}-S_{F} S_{\text {mean }}\right)-H_{c, i}\left(S_{\text {worst }}-\left|S_{\text {old }, i j}\right|\right)
$$

Student phase: The hyper-chaotic sequences are introduced instead of random numbers to increase the population's diversity of learners. It is worthy to note that the standard distribution fails to reach a satisfactory level of ergodicity.

The new learning process is described as follows: If $X_{j}$ is better than $X_{k}$

$$
S_{\text {new }, i}=S_{o l d, i}+H_{c, i}\left(S_{o l d, i}-S_{o l d, j}\right)+H_{c, i}\left(S_{o l d, k_{1}}-S_{o l d, k_{2}}\right)
$$

Else

$$
S_{\text {new }, i}=S_{o l d, i}+H_{c, i}\left(S_{o l d, i}-S_{o l d, j}\right)+H_{c, i}\left(S_{o l d, k_{2}}-S_{o l d, k_{1}}\right)
$$

\subsection{New Hyperchaotic Perturbation Strategy}

A new hyperchaotic perturbation strategy is embedded in the original TLBO algorithm to improve the obtained solution further. This perturbation strategy is mathematically formulated as follows:

$$
V_{j}=S_{j, \text { new }}\left(1+H c_{j}\right)
$$

The step size of the hyperchaotic perturbation affects its search capability. In other words, a significant step leads to avoiding local optima by performing a considerable jump. In contrast, a small step is beneficial to look for a good solution and boost exploitation ability.

The pseudo-code of the HTLBO algorithm is summarized in Algorithm 1. 


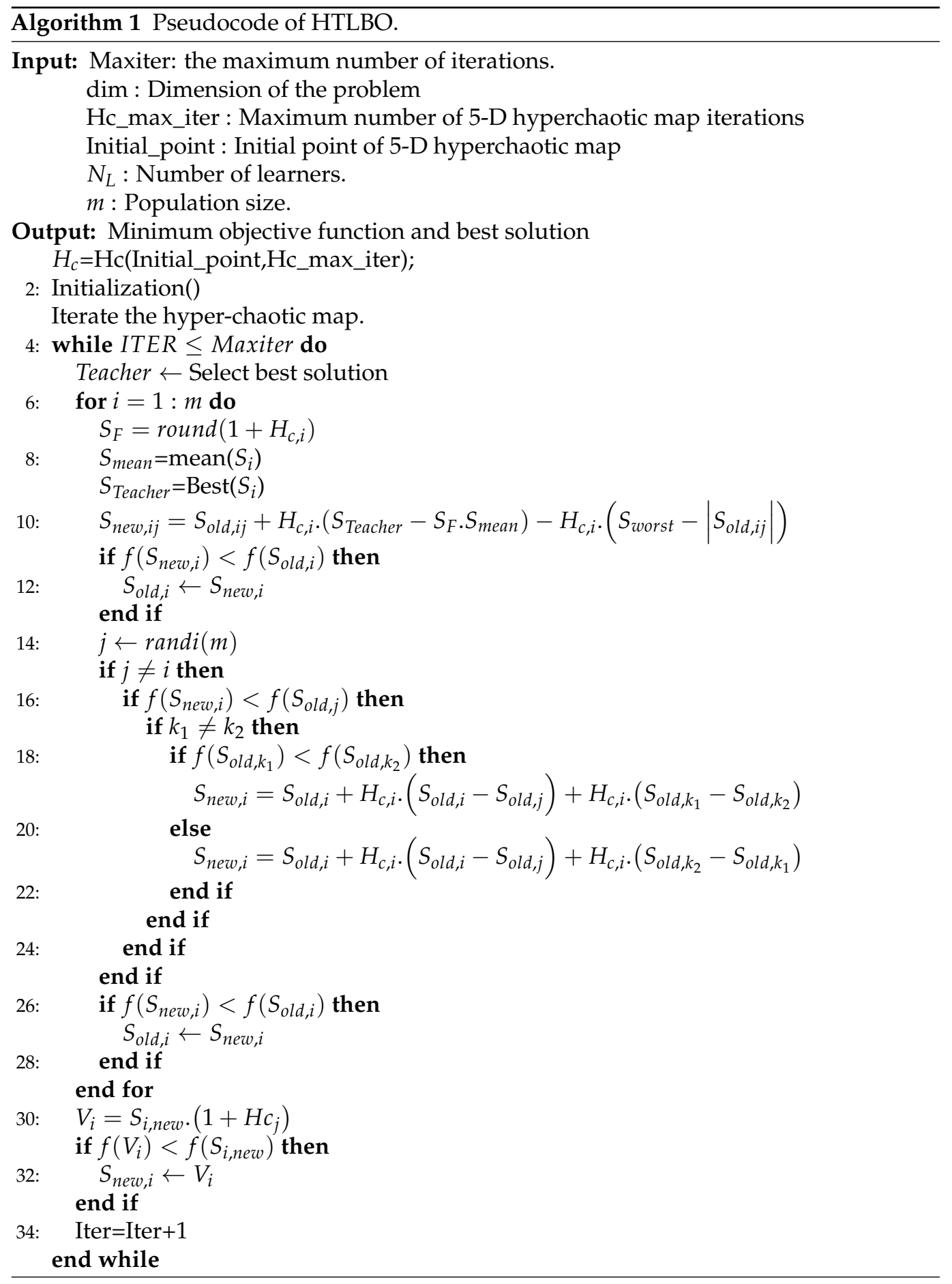

\section{Numerical Results from Benchmark Testing}

The proposed HTLBO and the comparative algorithms are implemented using MATLAB 2017a, and a PC with 4 GB of RAM and a processor I7 with $2.3 \mathrm{GHz}$. The performance of the HTLBO was assessed against popular optimization algorithms such as PSO, FA, DE, and standard TLBO. To achieve a fair comparison, all used algorithms were executed for 30 independent runs under 100,000 function evaluations and a population size of 40 for each benchmark function.

In this paper, the effectiveness of the used optimization algorithms are evaluated via a set of benchmark functions with different characteristics listed in Tables 1-3, where Dim and range indicates the dimension and the boundary of the search space, respectively. In line with this process, 26 benchmark functions are involved from the literature to achieve a fair 
comparison. The used benchmark functions are divided into three types: unimodal, multimodal, and rotated problems. The first category of the test functions $\left(f_{1}-f_{8}\right)$ is characterized by only one global optimum (i.e., no local optima), and as a consequence, they are used to benchmark the exploitation propriety. The second category, namely multimodal test functions $\left(f_{9}-f_{18}\right)$, are suitable for examination of the exploration characteristic of a given optimization algorithm since they have several local optima. The third category $\left(f_{19}-f_{26}\right)$ is composed of the rotated unimodal and multimodal functions. The latter category was chosen for its similarity to a real-life problem and its capability to judge the balance between exploitation and exploration propriety of a given optimization algorithm. The statistical results including the mean of the best (Mean) and the standard deviation (StdDev) are reported in Tables 4-6.

Table 1. Description of unimodal functions.

\begin{tabular}{lccc}
\hline Name & Formula & Range & Acceptance \\
\hline Sphere & $f_{1}(x)=\sum_{i=1}^{D} x_{i}^{2}$ & {$[-100,100]$} & $1 e-6$ \\
Schwefel 1.2 & $f_{2}(x)=\sum_{i=1}^{D}\left(\sum_{j=1}^{i} x_{j}\right)^{2}$ & {$[-100,100]$} & $1 e-6$ \\
Schwefel 2.21 & $f_{3}(x)=\max \left\{\left|x_{i}\right|, 1 \leq i \leq D\right\}$ & {$[-100,100]$} & $1 e-5$ \\
Scwefel 2.22 & $f_{4}(x)=\sum_{i=1}^{D}\left|x_{i}\right|+\prod_{i=1}^{D}\left|x_{i}\right|$ & {$[-10,10]$} & $1 e-6$ \\
Elliptic & $f_{5}(x)=\sum_{i=1}^{D}\left(10^{6}\right)^{i-1}$ & {$[-100,100]$} & $1 e-6$ \\
SumPower & $f_{6}(x)=\sum_{i=1}^{D}\left|x_{i}\right|^{(i+1)}$ & {$[-1,1]$} & $1 e-6$ \\
Quartic & $f_{7}(x)=\sum_{i=1}^{D} i x_{i}^{4}+$ random $(0,1)$ & {$[-1.28,1.28]$} & $1 e-2$ \\
Rosenbrock & $f_{8}(x)=\sum_{i=1}^{D-1}\left[100\left(x_{i}^{2}-x_{i+1}\right)^{2}+\left(x_{i}-1\right)^{2}\right]$ & {$[-5,10]$} & 50 \\
\hline
\end{tabular}

Table 2. Description of the multimodal functions.

\begin{tabular}{|c|c|c|c|}
\hline Name & Formula & Range & Acceptance \\
\hline Dixon-price & $f_{9}(x)=\left(x_{1}-1\right)^{2}+\sum_{i=1}^{D} i\left(2 x_{i}-x_{i-1}\right)^{2}$ & {$[-10,10]$} & 1 \\
\hline Zakharov & $f_{10}(x)=\sum_{i=1}^{D} x_{i}^{2}+\left(\sum_{i=1}^{D} 0.5 x_{i}\right)^{2}+\left(\sum_{i=1}^{n} 0.5 x_{i}\right)^{4}$ & {$[-10,10]$} & $1 e-6$ \\
\hline Rastrigin & $f_{11}(x)=\sum_{i=1}^{D}\left(x_{i}^{2}-10 \cos \left(2 \pi x_{i}+10\right)\right)$ & {$[-5.12,5.12]$} & $1 e-2$ \\
\hline Ackley & $f_{12}(x)=-20 \exp \left(-0.2 \sqrt{\frac{1}{D} \sum_{i=1}^{D} x_{i}^{2}}\right)-\exp \left(\frac{1}{D} \sum_{i=1}^{D} \cos \left(2 \pi x_{i}\right)\right)+20+e$ & {$[-32,32]$} & $1 e-2$ \\
\hline Griewank & $f_{13}(x)=\sum_{i=1}^{D} \frac{x_{i}^{2}}{4000}-\prod_{i=1}^{D} \cos \left(\frac{x_{i}}{\sqrt{i}}\right)+1$ & {$[-600,600]$} & $1 e-2$ \\
\hline \multirow[t]{2}{*}{ Penalized2 } & $f_{14}(x)=\frac{1}{10}\left[\sin ^{2}\left(3 \pi x_{1}\right)+\sum_{i=1}^{n-1}\left(x_{i}-1\right)^{2}\left(1+10 \sin ^{2}\left(3 \pi x_{i+1}\right)\right)\right.$ & {$[-50,50]$} & $8 e-1$ \\
\hline & $\left.+\left(x_{n}-1\right)^{2}\left(1+\sin ^{2}\left(2 \pi x_{n}\right)\right)\right]+\sum_{i=1}^{n} u\left(x_{i}, 5,100,4\right)$ & {$[-50,50]$} & \\
\hline Alpine & $f_{15}(x)=\sum_{i=1}^{D}\left|x_{i} \sin \left(x_{i}\right)+0.1 * x_{i}\right|$ & {$[-10,10]$} & $1 e-6$ \\
\hline NC Rastrigin & $f_{16}(x)=\sum_{i=1}^{D}\left(y_{i}^{2}-10 \cos \left(2 \pi y_{i}+10\right)\right)$ & {$[-5.12,5.12]$} & $1 e-2$ \\
\hline \multirow[t]{4}{*}{ Levy } & $f_{17}(x)=\sin ^{2}\left(\pi y_{1}\right)+\sum_{i=1}^{n-1}\left(y_{i}-1\right)^{2}\left(1+10 \sin ^{2}\left(\pi y_{i}+1\right)\right)$ & {$[-10,10]$} & $1 e+00$ \\
\hline & $+\left(y_{n}-1\right)^{2}\left(1+\sin ^{2}\left(2 \pi y_{n}\right)\right)$ & & \\
\hline & $y_{i}=1+\frac{1}{4}\left(x_{i}-1\right)$ & & \\
\hline & $y_{i}= \begin{cases}x_{i} & \left|x_{i}\right|<\frac{1}{2} \\
\frac{\operatorname{round}\left(2 x_{i}\right)}{2} & \left|x_{i}\right| \geq \frac{1}{2}\end{cases}$ & & \\
\hline \multirow[t]{2}{*}{ Weierstrass } & $f_{18}(x)=\sum_{i=1}^{D}\left(\sum_{k=0}^{k_{\max }}\left[a^{k} \cos \left(2 \pi b^{k}\left(x_{i}+0.5\right)\right)\right]\right)-D \sum_{k=0}^{k_{\max }}\left[a^{k}\right.$ & {$[-0.5,0.5]$} & $1 e-2$ \\
\hline & $\left.\cos \left(2 \pi b^{k} \times 0.5\right)\right] \quad a=0.5 \quad b=3 \quad k_{\max }=20$ & & \\
\hline
\end{tabular}


Table 3. Description of rotated functions.

\begin{tabular}{lccc}
\hline Name & Formula & Range & Acceptance \\
\hline Category 1: Rotated unimodal functions & $f_{19}(x)=f_{1}(z), z=M * x$ & {$[-100,100]$} & $1 e-6$ \\
Rotated sphere & $f_{20}(x)=f_{2}(z), z=M * x$ & {$[-100,100]$} & $1 e-6$ \\
Rotated schwefel 1.2 & $f_{21}(x)=f_{5}(z), z=M * x$ & {$[-100,100]$} & $1 e-6$ \\
Rotated elliptic & $f_{22}(x)=f_{6}(z), z=M * x$ & {$[-1,1]$} & $1 e-6$ \\
Rotated sum power & & $1 e-2$ \\
Category 2: Rotated multimodal functions & $f_{23}(x)=f_{13}(z), z=M * x$ & {$[-5.12,5.12]$} & $1 e-2$ \\
Rotated rastrigin & $f_{24}(x)=f_{14}(z), z=M * x$ & {$[-32,32]$} & $1 e-2$ \\
Rotated ackley & $f_{25}(x)=f_{15}(z), z=M * x$ & {$[-600,600]$} & $1 e-2$ \\
Rotated griewank & $f_{26}(x)=f_{19}(z), z=M * x$ & {$[-5.12,5.12]$} & \\
Rotated NC rastrigin & &
\end{tabular}

\subsection{Solution Accuracy of the Used Algorithms}

Table 4 shows that HTLBO outperforms all competitive algorithms in terms of the mean of the best solution and the standard deviation (SD) for the unimodal functions $f_{1}$, $f_{2}, f_{3}, f_{4}, f_{5}$ and $f_{7}$. For the last unimodal function $f_{8}$, the PSO algorithm provides the best results.

Table 4. Results comparisons of unimodal test functions on 30-dimensional functions $f_{1}-f_{8}$.

\begin{tabular}{|c|c|c|c|c|c|c|}
\hline Function & & PSO & FA & DE & TLBO & HTLBO \\
\hline \multirow{2}{*}{$f_{1}$} & Mean & $1.364 \mathrm{e}-86$ & $1.099 \mathrm{e}-02$ & $2.027 \mathrm{e}-36$ & $1.999 \mathrm{e}-227$ & $0.000 \mathrm{e}+00$ \\
\hline & StdDev & $7.471 \mathrm{e}-86$ & $5.991 \mathrm{e}-02$ & $1.560 \mathrm{e}-36$ & $0.000 \mathrm{e}+00$ & $0.000 \mathrm{e}+00$ \\
\hline \multirow{2}{*}{$f_{2}$} & Mean & $3.017 \mathrm{e}-08$ & $2.658 \mathrm{e}+02$ & $1.452 \mathrm{e}+04$ & $3.249 \mathrm{e}-52$ & $0.000 \mathrm{e}+00$ \\
\hline & StdDev & $1.652 \mathrm{e}-07$ & $9.105 e+02$ & $2.658 \mathrm{e}+03$ & $1.399 \mathrm{e}-52$ & $0.000 \mathrm{e}+00$ \\
\hline \multirow[t]{2}{*}{$f_{3}$} & Mean & $5.111 \mathrm{e}-05$ & $1.753 e+01$ & $7.2631 \mathrm{e}-03$ & $6.449 \mathrm{e}-92$ & $0.000 \mathrm{e}+00$ \\
\hline & StdDev & $2.799 \mathrm{e}-04$ & $8.096 \mathrm{e}+00$ & $1.373 \mathrm{e}-03$ & $3.911 \mathrm{e}-92$ & $0.000 e+00$ \\
\hline \multirow{2}{*}{$f_{4}$} & Mean & $8.933 \mathrm{e}-06$ & $8.206 \mathrm{e}-03$ & $2.424 \mathrm{e}-22$ & $2.69 \mathrm{e}-113$ & $0.000 \mathrm{e}+00$ \\
\hline & StdDev & $4.888 \mathrm{e}-05$ & $4.144 \mathrm{e}-02$ & $8.803 \mathrm{e}-23$ & $2.468 \mathrm{e}-113$ & $0.000 \mathrm{e}+00$ \\
\hline \multirow[t]{2}{*}{$f_{5}$} & Mean & $5.403 e-75$ & $2.997 \mathrm{e}+03$ & $4.580 \mathrm{e}-33$ & $5.420 \mathrm{e}-223$ & $0.000 \mathrm{e}+00$ \\
\hline & StdDev & $2.959 \mathrm{e}-74$ & $1.404 \mathrm{e}+04$ & $5.019 \mathrm{e}-33$ & $0.000 \mathrm{e}+00$ & $0.000 \mathrm{e}+00$ \\
\hline \multirow{2}{*}{$f_{6}$} & Mean & $8.171 \mathrm{e}-240$ & $8.5579 \mathrm{e}-12$ & $1.509 \mathrm{e}-115$ & $0.000 e+00$ & $0.000 \mathrm{e}+00$ \\
\hline & StdDev & $0.00 \mathrm{e}+00$ & $4.6162 \mathrm{e}-11$ & $7.084 \mathrm{e}-115$ & $0.000 \mathrm{e}+00$ & $0.000 \mathrm{e}+00$ \\
\hline \multirow[t]{2}{*}{$f_{7}$} & Mean & $7.267 \mathrm{e}-04$ & $9.342 \mathrm{e}-03$ & $9.379 \mathrm{e}-03$ & $2.339 \mathrm{e}-04$ & $3.215 \mathrm{e}-05$ \\
\hline & StdDev & $9.650 \mathrm{e}-04$ & $3.364 \mathrm{e}-03$ & $2.103 \mathrm{e}-03$ & 6.353e-05 & $2.490 \mathrm{e}-05$ \\
\hline \multirow{2}{*}{$f_{8}$} & Mean & $1.503 e+00$ & $4.430 \mathrm{e}+00$ & $2.693 e+01$ & $1.896 \mathrm{e}+01$ & $2.766 \mathrm{e}+01$ \\
\hline & StdDev & $4.819 e+00$ & $3.636 \mathrm{e}+01$ & $1.046 \mathrm{e}+01$ & $6.773 \mathrm{e}-01$ & $3.891 \mathrm{e}-01$ \\
\hline
\end{tabular}

Table 5 indicates that all algorithms give practically similar results for functions $f_{9}$. For functions $f_{10}, f_{11}, f_{13}, f_{15}, f_{16}$ and $f_{18}$ the proposed algorithm is the best among all used algorithms. Its final solutions are the theoretical global optimum. For functions, $f_{12}$ all used algorithms fail to reach the global optimum, but the proposed algorithm still ranked first. For function $f_{14}$ and $f_{17}$, DE give better performance among all algorithms followed by PSO algorithm.

Table 6 pinpoint that the proposed HTLBO outperform all comparative algorithms and reach the theoretical global optimum for the unimodal rotated problem $f_{19}, f_{20}, f_{21}$, $f_{22}, f_{23}, f_{25}$ and $f_{26}$. Despite all used algorithms failing to attain the global optimum for the function $f_{24}$, HTLBO algorithm provides a promising result. 
Table 5. Results for 30-dimensional multimodal functions $f_{9}-f_{18}$.

\begin{tabular}{|c|c|c|c|c|c|c|}
\hline Function & & PSO & FA & $\mathrm{DE}$ & TLBO & HTLBO \\
\hline \multirow{2}{*}{$f_{9}$} & Mean & $6.666 \mathrm{e}-01$ & 8.817e-01 & $6.666 \mathrm{e}-01$ & $6.666 \mathrm{e}-01$ & $6.666 \mathrm{e}-01$ \\
\hline & StdDev & $5.492 \mathrm{e}-15$ & $6.647 \mathrm{e}-01$ & $4.659 \mathrm{e}-06$ & $5.961 \mathrm{e}-16$ & $4.190 \mathrm{e}-10$ \\
\hline \multirow{2}{*}{$f_{10}$} & Mean & $2.847 \mathrm{e}-12$ & $4.380 \mathrm{e}-01$ & $1.272 \mathrm{e}+02$ & $5.909 \mathrm{e}-30$ & $0.000 \mathrm{e}+00$ \\
\hline & StdDev & $1.559 \mathrm{e}-11$ & $3.969 \mathrm{e}-01$ & $2.686 \mathrm{e}+01$ & $1.891 \mathrm{e}-29$ & $0.000 \mathrm{e}+00$ \\
\hline \multirow[t]{2}{*}{$f_{11}$} & Mean & $2.193 e+01$ & $5.377 \mathrm{e}+01$ & $2.663 e+01$ & $6.786 \mathrm{e}+00$ & $0.000 \mathrm{e}+00$ \\
\hline & StdDev & $4.435 \mathrm{e}+00$ & $2.019 \mathrm{e}+01$ & $6.195 \mathrm{e}+00$ & $2.257 \mathrm{e}+00$ & $0.000 \mathrm{e}+00$ \\
\hline \multirow{2}{*}{$f_{12}$} & Mean & $3.218 \mathrm{e}-01$ & $6.925 \mathrm{e}-03$ & 7.993e-15 & $4.441 \mathrm{e}-15$ & 8.882e-16 \\
\hline & StdDev & $5.550 \mathrm{e}-01$ & $3.615 e-02$ & $0.000 \mathrm{e}+00$ & $0.000 \mathrm{e}+00$ & $0.000 \mathrm{e}+00$ \\
\hline \multirow{2}{*}{$f_{13}$} & Mean & $9.836 \mathrm{e}-13$ & $2.216 \mathrm{e}-02$ & $0.000 e+00$ & $0.000 e+00$ & $0.000 e+00$ \\
\hline & StdDev & $5.387 \mathrm{e}-12$ & $7.732 \mathrm{e}-02$ & $0.000 e+00$ & $0.000 e+00$ & $0.000 \mathrm{e}+00$ \\
\hline \multirow[t]{2}{*}{$f_{14}$} & Mean & $1.750 \mathrm{e}-10$ & $2.677 \mathrm{e}-03$ & $1.349 \mathrm{e}-32$ & $3.245 \mathrm{e}-03$ & $3.511 \mathrm{e}-01$ \\
\hline & StdDev & $9.585 \mathrm{e}-10$ & $4.963 \mathrm{e}-03$ & $5.567 \mathrm{e}-48$ & $1.777 \mathrm{e}-02$ & $1.36-\mathrm{e}-01$ \\
\hline \multirow[t]{2}{*}{$f_{15}$} & Mean & $4.135 \mathrm{e}-04$ & $1.856 \mathrm{e}-03$ & $3.201 \mathrm{e}-04$ & $4.143 e-114$ & $0.000 \mathrm{e}+00$ \\
\hline & StdDev & $1.138 \mathrm{e}-03$ & $8.506 \mathrm{e}-03$ & $3.889 \mathrm{e}-04$ & $1.279 \mathrm{e}-113$ & $0.000 \mathrm{e}+00$ \\
\hline \multirow[t]{2}{*}{$f_{16}$} & Mean & $2.203 e+01$ & $5.633 e+01$ & $2.424 \mathrm{e}+01$ & $1.256 \mathrm{e}+01$ & $0.000 \mathrm{e}+00$ \\
\hline & StdDev & $7.490 \mathrm{e}+00$ & $1.930 \mathrm{e}+01$ & $1.052 \mathrm{e}+00$ & $2.824 \mathrm{e}+00$ & $0.000 \mathrm{e}+00$ \\
\hline \multirow{2}{*}{$f_{17}$} & Mean & $3.625 e-02$ & $2.08 \mathrm{e}-01$ & $1.499 \mathrm{e}-32$ & $2.506 \mathrm{e}-01$ & $4.720 \mathrm{e}-01$ \\
\hline & StdDev & $1.379 \mathrm{e}-01$ & $4.571 \mathrm{e}-01$ & $1.113 e-47$ & $6.814 \mathrm{e}-02$ & $1.737 \mathrm{e}-01$ \\
\hline \multirow[t]{2}{*}{$f_{18}$} & Mean & $1.682 \mathrm{e}+00$ & $4.908 \mathrm{e}-02$ & $0.000 e+00$ & $0.000 e+00$ & $0.000 \mathrm{e}+00$ \\
\hline & StdDev & $6.632 \mathrm{e}-01$ & $2.192 \mathrm{e}-01$ & $0.000 e+00$ & $0.000 e+00$ & $0.000 \mathrm{e}+00$ \\
\hline
\end{tabular}

\subsection{Comparison of Convergence}

The mean number of evaluations and success rate over 30 independent runs is calculated to compare all used algorithms in convergence speed. As for the unimodal functions, Table 7 shows that the HTLBO required a lesser number of functions evaluations to reach the final solutions and had a higher success rate than all comparative algorithms for all unimodal functions.

The convergence graph of the used algorithms for $f_{1}, f_{3}, f_{5}$ and $f_{8}$ functions are drawn in Figure 3. These figures indicate that the convergence process of the HTLBO algorithm is quicker than all comparative algorithms.

Table 6. Results for 30-dimensional rotated functions $f_{19}-f_{26}$.

\begin{tabular}{|c|c|c|c|c|c|c|}
\hline Function & & PSO & FA & DE & TLBO & HTLBO \\
\hline \multirow{2}{*}{$f_{19}$} & Mean & $6.962 \mathrm{e}-18$ & $1.086 \mathrm{e}-02$ & $2.981 \mathrm{e}-36$ & $1.912 \mathrm{e}-226$ & $0.000 \mathrm{e}+00$ \\
\hline & StdDev & $3.813 \mathrm{e}-17$ & $5.918 \mathrm{e}-02$ & $2.447 \mathrm{e}-36$ & $0.000 \mathrm{e}+00$ & $0.000 e+00$ \\
\hline \multirow{2}{*}{$f_{20}$} & Mean & $2.329 \mathrm{e}-08$ & $4.419 \mathrm{e}+02$ & $1.434 \mathrm{e}+04$ & $1.223 e-51$ & $0.000 e+00$ \\
\hline & StdDev & $1.275 \mathrm{e}-07$ & $1.261 \mathrm{e}+03$ & $2.720 \mathrm{e}+03$ & $3.246 \mathrm{e}-51$ & $0.000 e+00$ \\
\hline \multirow[t]{2}{*}{$f_{21}$} & Mean & $7.726 \mathrm{e}-30$ & $4.563 e+02$ & $3.093 e-33$ & $3.406 \mathrm{e}-221$ & $0.000 e+00$ \\
\hline & StdDev & $4.232 \mathrm{e}-29$ & $2.481 \mathrm{e}+03$ & $2.386 \mathrm{e}-33$ & $0.000 \mathrm{e}+00$ & $0.000 e+00$ \\
\hline \multirow{3}{*}{$f_{22}$} & Mean & $1.764 \mathrm{e}-241$ & $7.768 \mathrm{e}-11$ & $1.733 \mathrm{e}-116$ & $0.000 \mathrm{e}+00$ & $0.000 e+00$ \\
\hline & StdDev & $0.000 \mathrm{e}+00$ & $4.251 \mathrm{e}-10$ & 7.474e-116 & $0.000 \mathrm{e}+00$ & $0.000 e+00$ \\
\hline & SEM & $0.000 \mathrm{e}+00$ & $7.762 \mathrm{e}-11$ & $1.364 \mathrm{e}-116$ & $0.000 e+00$ & $0.000 e+00$ \\
\hline \multirow[t]{2}{*}{$f_{23}$} & Mean & $3.691 \mathrm{e}+01$ & $4.945 \mathrm{e}+01$ & $2.525 \mathrm{e}+01$ & $3.658 \mathrm{e}+00$ & $0.000 \mathrm{e}+00$ \\
\hline & StdDev & $9.232 \mathrm{e}+00$ & $1.224 \mathrm{e}+01$ & $8.129 e+00$ & $3.457 \mathrm{e}+00$ & $0.000 e+00$ \\
\hline \multirow{2}{*}{$f_{24}$} & Mean & $1.258 \mathrm{e}-01$ & $5.598 \mathrm{e}-03$ & $8.112 \mathrm{e}-15$ & $4.441 \mathrm{e}-15$ & $8.882 \mathrm{e}-16$ \\
\hline & StdDev & $3.915 \mathrm{e}-01$ & $2.874 \mathrm{e}-02$ & $6.486 \mathrm{e}-16$ & $0.000 \mathrm{e}+00$ & $0.000 e+00$ \\
\hline \multirow[t]{2}{*}{$f_{25}$} & Mean & $5.398 \mathrm{e}-02$ & $2.272 \mathrm{e}-02$ & $0.000 \mathrm{e}+00$ & $0.000 \mathrm{e}+00$ & $0.000 e+00$ \\
\hline & StdDev & $1.704 \mathrm{e}-14$ & $8.855 \mathrm{e}-02$ & $0.000 \mathrm{e}+00$ & $0.000 \mathrm{e}+00$ & $0.000 e+00$ \\
\hline \multirow[t]{2}{*}{$f_{26}$} & Mean & $2.123 e+01$ & $6.834 \mathrm{e}+01$ & $2.401 \mathrm{e}+01$ & $1.423 \mathrm{e}+01$ & $0.000 e+00$ \\
\hline & StdDev & $6.334 \mathrm{e}+00$ & $3.125 \mathrm{e}+01$ & $1.753 \mathrm{e}+00$ & $2.996 \mathrm{e}+00$ & $0.000 e+00$ \\
\hline
\end{tabular}



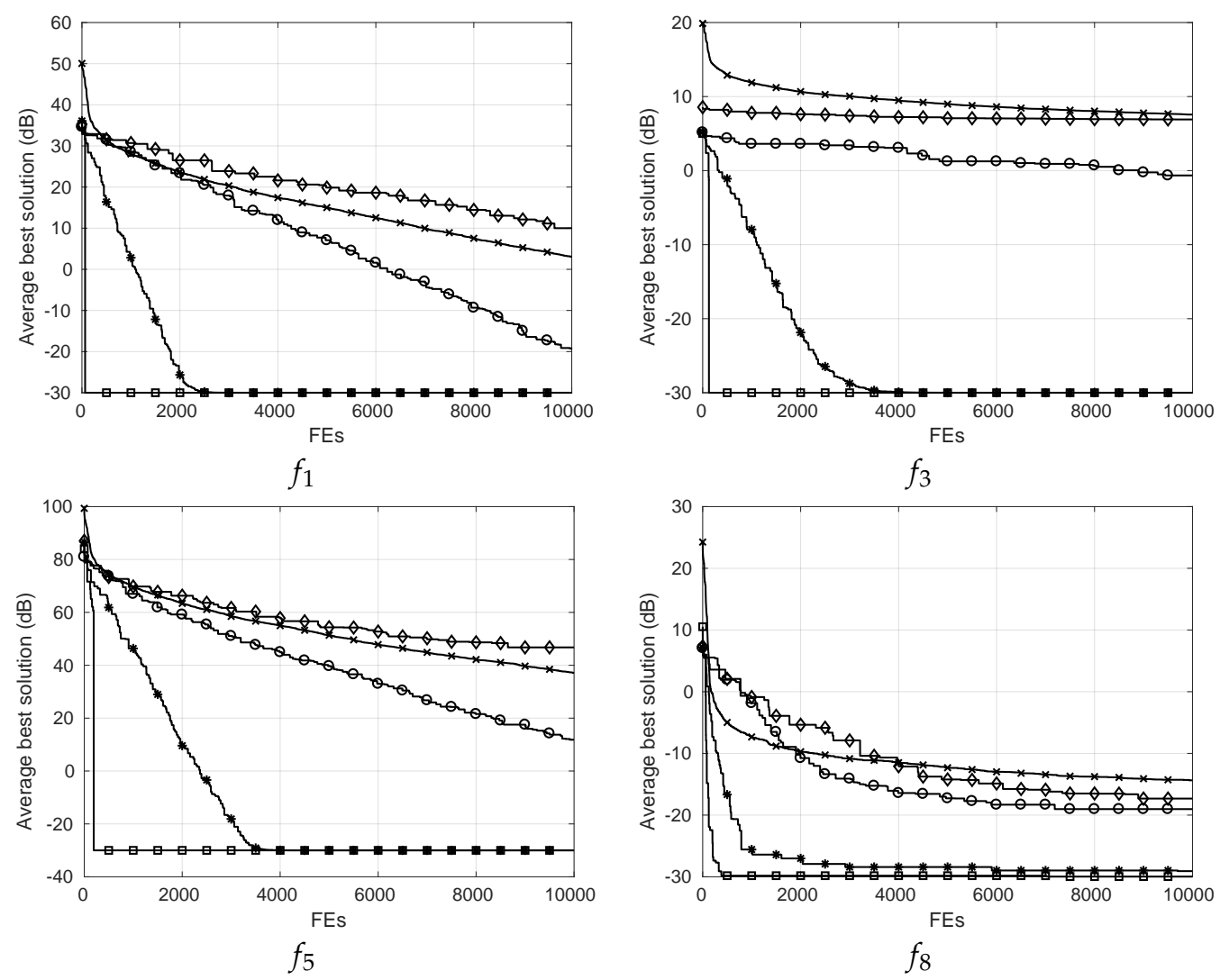

Figure 3. Convergence performance of different algorithms on the unimodal functions. DE [o], TLBO $[*]$, PSO $[\times]$, HITLBO $[\square]$, and FA $[\diamond]$.

Table 7. MeanFes and SR by all used algorithms for unimodal functions.

\begin{tabular}{|c|c|c|c|c|c|c|}
\hline Function & & PSO & FA & $\mathrm{DE}$ & TLBO & HTLBO \\
\hline \multirow[t]{2}{*}{$f_{1}$} & MeanFes & 999.9 & $77,663.185$ & $26,630.733$ & 4790.667 & 189.967 \\
\hline & SR (\%) & $100 \%$ & $90 \%$ & $100 \%$ & $100 \%$ & $100 \%$ \\
\hline \multirow[t]{2}{*}{$f_{2}$} & MeanFes & 4077.633 & NAN & NAN & $19,133.933$ & 169.533 \\
\hline & SR (\%) & $100 \%$ & $0 \%$ & $0 \%$ & $100 \%$ & $100 \%$ \\
\hline \multirow[t]{2}{*}{$f_{3}$} & MeanFes & 3574.345 & NAN & NAN & 8654.633 & 190.8 \\
\hline & SR (\%) & $96.6667 \%$ & $0 \%$ & $0 \%$ & $100 \%$ & $100 \%$ \\
\hline \multirow[t]{2}{*}{$f_{4}$} & MeanFes & 1225.448 & $97,054.565$ & $34,492.6$ & 7245.433 & 252.033 \\
\hline & SR (\%) & $96.667 \%$ & $76.667 \%$ & $100 \%$ & $100 \%$ & $100 \%$ \\
\hline \multirow[t]{2}{*}{$f_{5}$} & MeanFes & 1324.767 & $98,660.471$ & $34,655.733$ & 6494.967 & 243.167 \\
\hline & SR (\%) & $100 \%$ & $56.667 \%$ & $100 \%$ & $100 \%$ & $100 \%$ \\
\hline \multirow[t]{2}{*}{$f_{6}$} & MeanFes & 261.567 & $11,975.433$ & 5106.2 & 1109.3 & 157.833 \\
\hline & SR (\%) & $100 \%$ & $100 \%$ & $100 \%$ & $100 \%$ & $100 \%$ \\
\hline \multirow[t]{2}{*}{$f_{7}$} & MeanFes & 1903.667 & $77,271.833$ & $83,476.05$ & 4746.3 & 594 \\
\hline & SR (\%) & $100 \%$ & $60 \%$ & $66.6667 \%$ & $100 \%$ & $100 \%$ \\
\hline \multirow[t]{2}{*}{$f_{8}$} & MeanFes & 527.5667 & $46,165.05$ & $21,700.1034$ & 1593 & 210.5667 \\
\hline & SR (\%) & $100 \%$ & $66.6667 \%$ & $96.6667 \%$ & $100 \%$ & $100 \%$ \\
\hline
\end{tabular}


Table 8 shows the obtained mean number of functions evaluations (MeanFes) and mean success rate over 30 independent runs by used algorithms on the multimodal test functions. The lesser values of mean number of functions evaluations and the associated success rate are shown in bold. The results demonstrate that the proposed HTLBO required less MeanFes and had a higher success rate than all comparative algorithms for test functions $f_{10}, f_{11}, f_{12}, f_{13}, f_{15}, f_{16}$ and $f_{18}$. Moreover, the proposed technique is ranked second for the test functions $f_{9}, f_{14}$ and $f_{17}$.

The convergence graph of the used algorithms for multimodal functions $f_{11}, f_{14}, f_{19}$ and $f_{20}$, is shown in Figure 4 . It can be observed from Figure 4 that the convergence speed of the proposed HTLBO algorithm is the quickest of all comparative algorithms.

The Mean number of function evaluations (MeanFes) and success ratio of each algorithm for 30-dimensional rotated benchmark functions are listed in Table 9. The mean number of function evaluations is recorded when the required conditions are reached. If the acceptance criterion is not attained after 30 runs, the mean FEs is expressed as ' $\mathrm{NaN}$,' and the success rate is $0 \%$. As we can see from Table 9 , the proposed technique exhibits the best results in terms of the MeanFes and success rate for all used benchmark functions.

Table 8. MeanFes and SR by all used algorithms for multimodal functions.

\begin{tabular}{|c|c|c|c|c|c|c|}
\hline Function & & PSO & FA & $\mathrm{DE}$ & TLBO & HTLBO \\
\hline \multirow[t]{2}{*}{$f_{9}$} & MeanFes & 559.067 & $55,895.286$ & $21,590.533$ & 2192.367 & 654.033 \\
\hline & SR (\%) & $100 \%$ & $93.3333 \%$ & $100 \%$ & $100 \%$ & $100 \%$ \\
\hline \multirow[t]{2}{*}{$f_{10}$} & MeanFes & 3151.467 & NAN & NAN & $36,511.7$ & 304.9 \\
\hline & SR (\%) & $100 \%$ & $0 \%$ & $0 \%$ & $100 \%$ & $100 \%$ \\
\hline \multirow[t]{2}{*}{$f_{11}$} & MeanFes & NAN & NAN & 26,862 & NAN & 173.6 \\
\hline & SR (\%) & $0 \%$ & $0 \%$ & $6.667 \%$ & $0 \%$ & $100 \%$ \\
\hline \multirow[t]{2}{*}{$f_{12}$} & MeanFes & 570.318 & $55,075.069$ & $18,976.033$ & 3558.1 & 175.933 \\
\hline & SR (\%) & $73.333 \%$ & $96.667 \%$ & $100 \%$ & $100 \%$ & $100 \%$ \\
\hline \multirow[t]{2}{*}{$f_{13}$} & MeanFes & 708.9 & $53,596.045$ & $19,370.367$ & 3457.667 & 178.067 \\
\hline & SR $(\%)$ & $100 \%$ & $73.333 \%$ & $100 \%$ & $100 \%$ & $100 \%$ \\
\hline \multirow[t]{2}{*}{$f_{14}$} & MeanFes & 403 & $37,067.3$ & $11,515.7667$ & 4510.2333 & 1608.6667 \\
\hline & SR (\%) & $100 \%$ & $100 \%$ & $100 \%$ & $100 \%$ & $100 \%$ \\
\hline \multirow[t]{2}{*}{$f_{15}$} & MeanFes & 1000.5 & $92,849.8636$ & 86,967 & 6719.3333 & 228.433 \\
\hline & SR $(\%)$ & $86.6667 \%$ & $73.3333 \%$ & $3.3333 \%$ & $100 \%$ & $100 \%$ \\
\hline \multirow[t]{2}{*}{$f_{16}$} & MeanFes & NAN & NAN & NAN & NAN & 229.433 \\
\hline & SR (\%) & $0 \%$ & $0 \%$ & $0 \%$ & $0 \%$ & $100 \%$ \\
\hline \multirow[t]{2}{*}{$f_{17}$} & MeanFes & 316.6 & $23,093.7241$ & 7834.1333 & 3225.1724 & 2012.8 \\
\hline & SR $(\%)$ & $100 \%$ & $96.6667 \%$ & $100 \%$ & $96.6667 \%$ & $100 \%$ \\
\hline \multirow[t]{2}{*}{$f_{18}$} & MeanFes & NAN & $72,856.8148$ & 26,098 & 5744.3 & 278.9 \\
\hline & SR (\%) & $0 \%$ & $90 \%$ & $100 \%$ & $100 \%$ & $100 \%$ \\
\hline
\end{tabular}



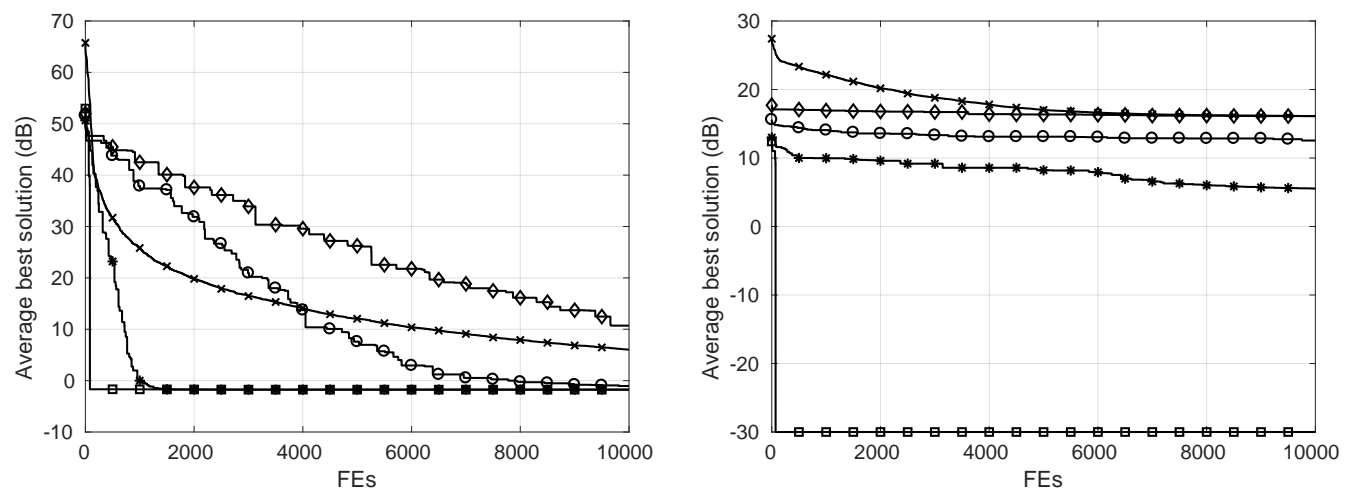

$f_{9}$
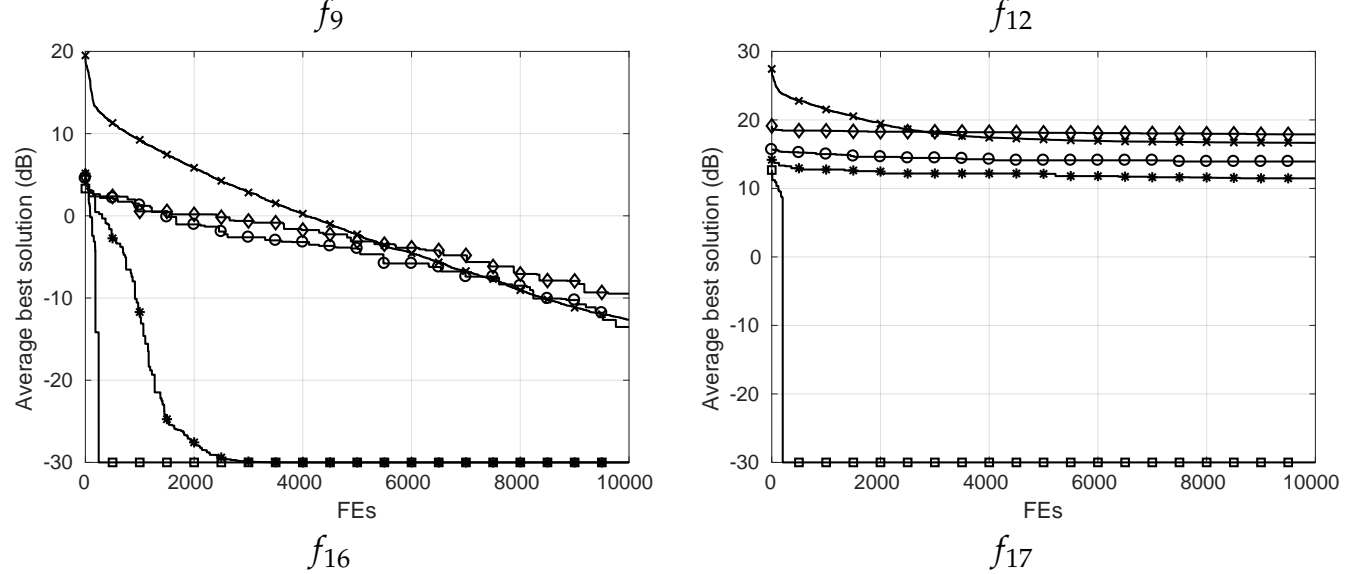

Figure 4. Convergence performance of different algorithms on the multimodal functions. DE $[0]$, TLBO $[*]$, PSO $[\times]$, HITLBO $[\square]$, and FA $[\diamond]$.

Additionally, a comparison of the convergence behavior of PSO, FA, FE, TLBO and HTLBO is presented in Figure 5, where $f_{19}, f_{21}, f_{25}$ and $f_{26}$ benchmark rotated functions are considered. These figures show that the proposed HTLBO exhibits a fast convergence performance due to its high diversity from the hyperchaotic map. Figure 5 reveals that the HTLBO outperforms the used comparative algorithms and converges to the optimal solution speedily.

It should be noted that an offset of 1e-3 has been added to the fitness values found by each algorithm. This is because some of the used algorithms reached the global optimum 0 . Consequently, in the convergence curves, the value $-30 \mathrm{~dB}$ indicates that the objective function equals the global optimum 0 .

\subsection{Statistical Tests}

In order to show the significance of the obtained results, we apply the Friedman rank test using the results given by Tables $4-6$. Table 10 presents the rank of the comparative algorithms. It is clear from the obtained results that $H T L B O$ outperforms all used algorithm, since it was ranked first. Original TLBO is ranked second according to obtained statistical test. 
Table 9. MeanFes and SR by all used algorithms for rotated test functions.

\begin{tabular}{|c|c|c|c|c|c|c|}
\hline Function & & PSO & FA & DE & TLBO & HTLBO \\
\hline \multirow[t]{2}{*}{$f_{19}$} & MeanFes & 1043.767 & $77,749.852$ & $26,667.367$ & 4785.8 & 246.567 \\
\hline & SR (\%) & $100 \%$ & $90 \%$ & $100 \%$ & $100 \%$ & $100 \%$ \\
\hline \multirow{2}{*}{$f_{20}$} & MeanFes & 4013.367 & NAN & NAN & $19,107.033$ & 260.233 \\
\hline & SR (\%) & $100 \%$ & $0 \%$ & $0 \%$ & $100 \%$ & $100 \%$ \\
\hline \multirow[t]{2}{*}{$f_{21}$} & MeanFes & 1315 & $98,377.067$ & $34,507.433$ & 6483.3 & 203.333 \\
\hline & SR (\%) & $100 \%$ & $50 \%$ & $100 \%$ & $100 \%$ & $100 \%$ \\
\hline \multirow[t]{2}{*}{$f_{22}$} & MeanFes & 248.1333 & 12415.1 & 5173.367 & 1100.9 & 189.233 \\
\hline & SR (\%) & $100 \%$ & $100 \%$ & $100 \%$ & $100 \%$ & $100 \%$ \\
\hline \multirow[t]{2}{*}{$f_{23}$} & MeanFes & NAN & NAN & NAN & NAN & 239.967 \\
\hline & SR (\%) & $0 \%$ & $0 \%$ & $0 \%$ & $0 \%$ & $100 \%$ \\
\hline \multirow[t]{2}{*}{$f_{24}$} & MeanFes & 554.593 & 55305.724 & $18,963.5$ & 3439.867 & 209.967 \\
\hline & SR (\%) & $90 \%$ & $96.667 \%$ & $100 \%$ & $100 \%$ & $100 \%$ \\
\hline \multirow[t]{2}{*}{$f_{25}$} & MeanFes & NAN & $52,346.783$ & $19,440.767$ & 3305.567 & 213.833 \\
\hline & SR (\%) & $0 \%$ & $76.667 \%$ & $100 \%$ & $100 \%$ & $100 \%$ \\
\hline \multirow[t]{2}{*}{$f_{26}$} & MeanFes & NAN & NAN & NAN & NAN & 170.7 \\
\hline & SR (\%) & $0 \%$ & $0 \%$ & $0 \%$ & $0 \%$ & $100 \%$ \\
\hline
\end{tabular}
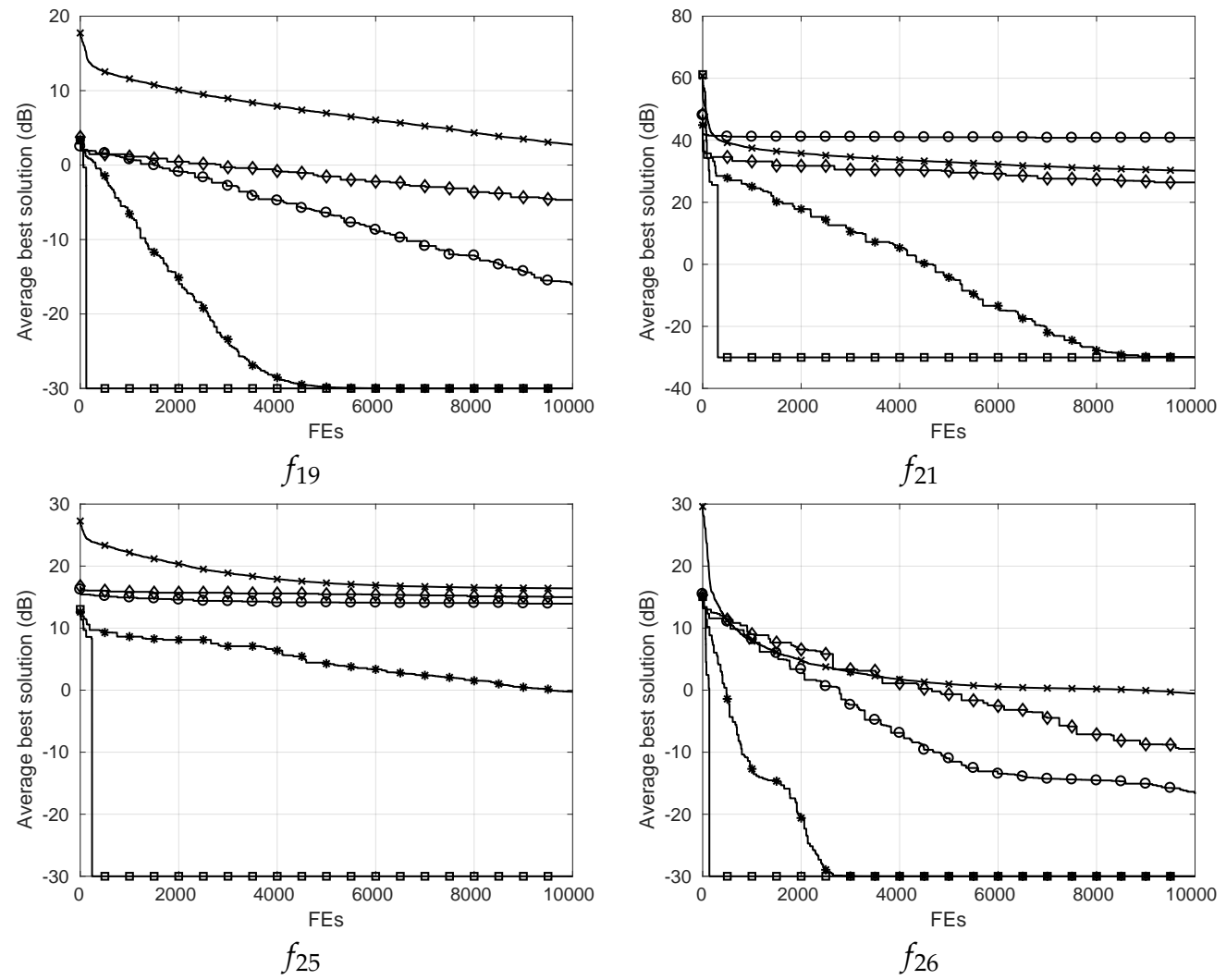

Figure 5. Convergence performance of different algorithms on the rotated functions. DE [o], TLBO $[*]$, PSO $[\times]$, HITLBO $[\square]$, and FA $[\diamond]$. 
Table 10. Algorithms ranking using Friedman test.

\begin{tabular}{llll}
\hline Algorithm & Friedman Value & Normalized Value & Rank \\
\hline PSO & 3.3846 & 2.022 & 4 \\
FA & 4.6923 & 2.804 & 5 \\
DE & 3.1923 & 1.908 & 3 \\
TLBO & 2.0577 & 1.229 & 2 \\
HTLBO & 1.6731 & 1.00 & 1 \\
\hline
\end{tabular}

\section{Power System Stability Design Problem Using the HTLBO}

\subsection{Study System Modeling}

The system of our study is a multimachine power system equipped with two controllers, which are Power System Stabilizer (PSS) and Static Var Compensator (SVC) shown in Figure 6. The object of this study is to design the controllers parameters using the proposed technique.

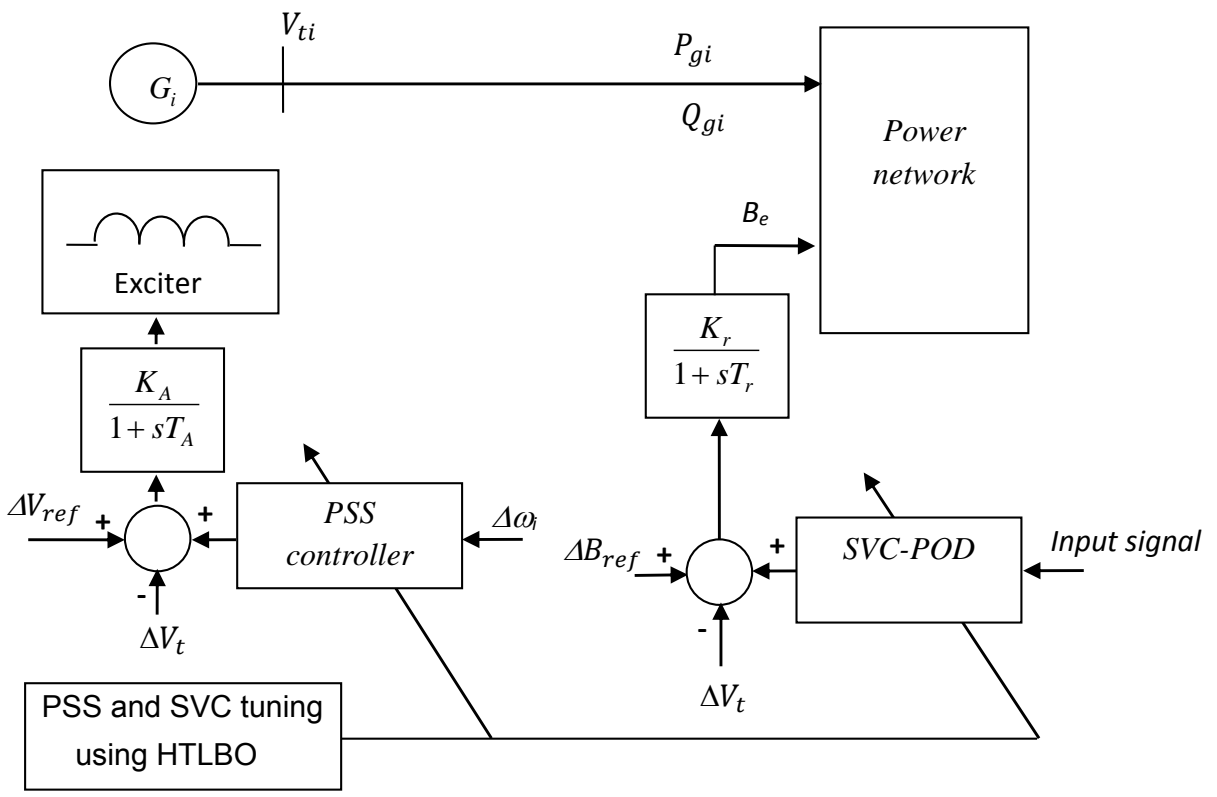

Figure 6. Power network with the proposed controllers.

\subsubsection{Test System}

Figure 7 shows the Western System Coordinating Council (WSCC) test system. WSCC is composed of three synchronous machines and nine buses. The parameters of the study system are illustrated in [9].

\subsubsection{Synchronous Machine Model}

The nonlinear dynamic of each machine can be described by the following third-order nonlinear differential-algebraic equations (DAE).

$$
\begin{gathered}
p \delta_{i}=\omega_{b}\left(\omega_{i}-1\right) \\
p \omega_{i}=\left(T_{m i}-T_{m i}-D_{i}\left(\omega_{i}-1\right)\right) / M_{i} \\
p E_{q i}^{\prime}=\left(E_{f d}-\left(x_{d}-x_{d}^{\prime}\right) i_{d i}-E_{q i}^{\prime}\right) / T_{d 0 i}^{\prime},
\end{gathered}
$$

where $\delta_{i}$ and $\omega_{i}$ are rotor angle and speed of the i-th machine, respectively. $\omega_{b}$ is the base frequency in rad/sec. $E_{q i}^{\prime}$ and $E_{f d}$ are internal and field voltages, respectively. $T_{m i}$ and $T_{e i}$ are mechanical and electric torques, respectively. $M_{i}, D_{i}$ and $T_{d 0 i}^{\prime}$ are the inertia constant, 
damping coefficient and open circuit field time constant of the machine, respectively. $x_{d}$ and $x_{d}^{\prime}$ are the $\mathrm{d}$-axis reactance and the d-axis transient reactance of the machine. $i_{d i}$ is the $\mathrm{d}$-axis armature current.

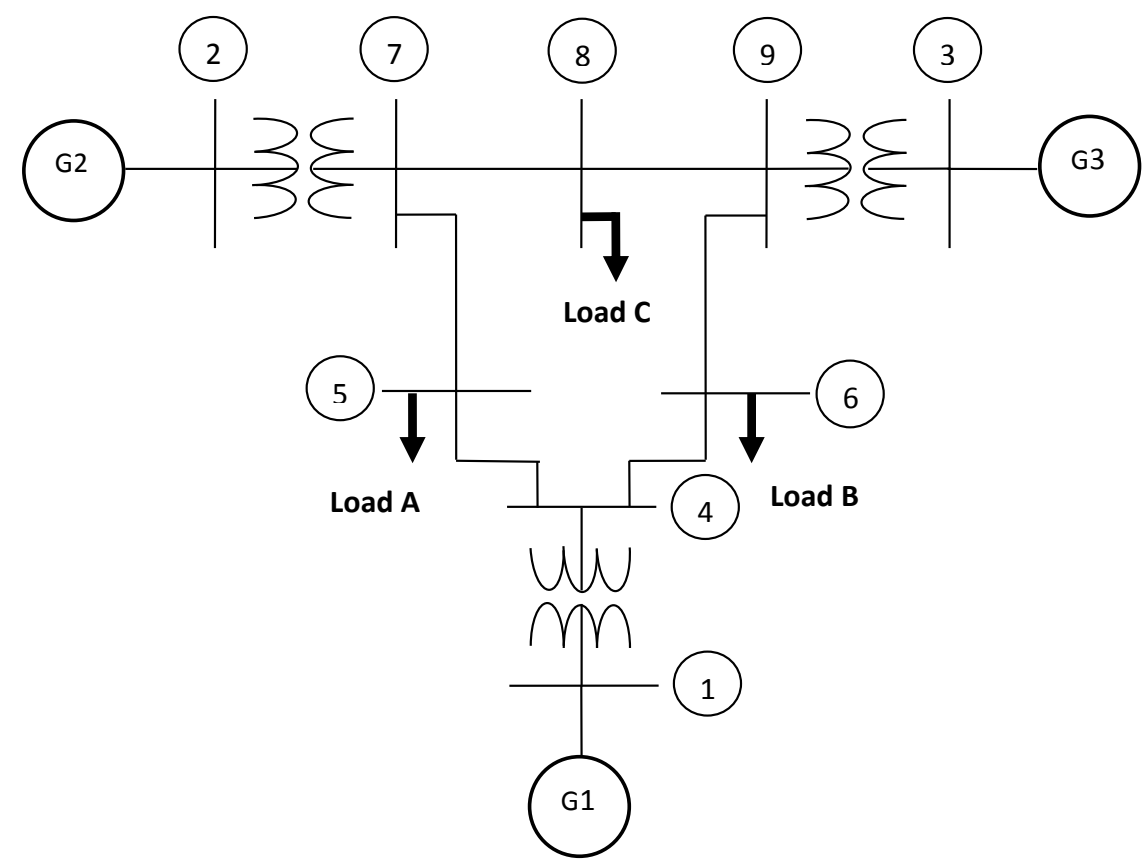

Figure 7. WSCC power system.

The electric torque can be written as follows.

$$
T_{e i}=E_{q i}^{\prime} i_{q i}+\left(x_{q i}-x_{d i}^{\prime}\right) i_{d i} i_{q i},
$$

where $x_{q i}$ and $i_{q i}$ are the q-axis reactance and the q-axis armature current of the $i$ th machine, respectively.

\subsubsection{Excitation System with PSS Controller}

The PSS acts through the excitation system to provide a control effect to the power system. The IEEE type-ST1 excitation system with PSS shown in Figure 8 is considered in this paper. It is described as follows.

$$
p E_{f d}=\left(K_{A}\left(V_{r e f}-V_{t}+U\right)-E_{f d}\right) / T_{A},
$$

where $K_{A}$ and $T_{A}$ are the amplifier gain and the amplifier time constants of the excitation system, respectively. $V_{r e f}$ and $V_{t}$ are reference and generator terminal voltages, respectively. The input signal of the $i$ th PSS is the normalized speed deviation of $\Delta \omega_{i}$. In contrast, the output is the supplementary stabilizing signal $U$. As given in the block diagram of Figure 8, the transfer function of the PSS is given below.

$$
U_{i}=K_{i}\left(\frac{s T_{\omega}}{1+s T_{\omega}}\right)\left(\frac{1+s T_{1 i}}{1+s T_{2 i}}\right)\left(\frac{1+s T_{3 i}}{1+s T_{4 i}}\right)
$$

In the previous equation, the washout block with a time constant $T_{\omega}$ is used as a high-pass filter to leave the signals in the range $0.2-2 \mathrm{~Hz}$ associated with rotor oscillation to pass without change. In general, it is in the range of 1-20 s. The two first-order lead-lag transfer functions are used to compensate for the phase lag between the PSS output and the control action, which is the electrical torque. 


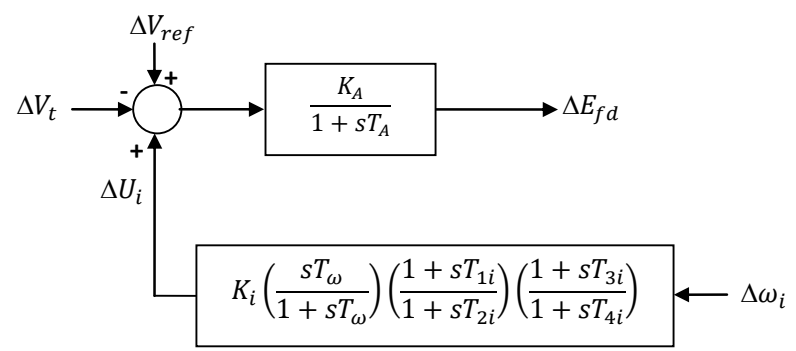

Figure 8. IEEE Type-ST1 excitation system with PSS.

\subsubsection{Structure of the SVC-Based POD Controller}

The SVC device used in this study is connected to the AC system via a setup transformer to keep the network voltage constantly at a set reference value by varying its effective reactance. As shown in Figure 9, the system is a thyristor-control reactor/fixed capacitor (TCR/FC). The firing angle of the thyristors controls the value of the effective reactance of the SVC, as written in the following equation:

$$
B_{v}=-\frac{(2 \pi-2 \alpha+\sin (2 \alpha))}{\pi X_{L}} ;-\frac{\pi}{2} \leq \alpha \leq \pi,
$$

where $X_{L}$ is the SVC fixed inductor reactance. The effective reactance is expressed in the following:

$$
X_{e}=X_{c} \frac{\pi / r_{x}}{\sin (2 \alpha)-2 \alpha+\pi\left(2-1 / r_{x}\right)}
$$

where $X_{e}=-1 / B_{e}$ and $r_{x}=X_{e} / X_{L}$.

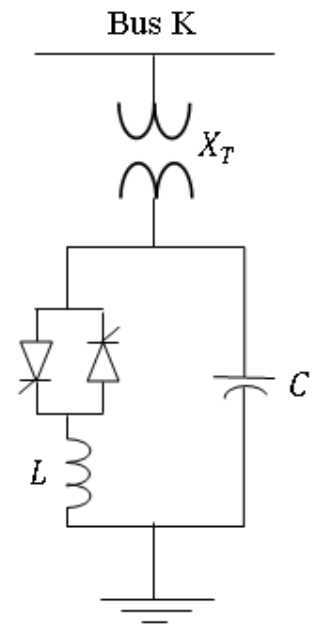

Figure 9. SVC power circuit.

The thyristor-control reactor is composed of a reactor in series with a bi-directional thyristor valve. The SVC reactance is modified using the TCR firing angle $\alpha$, which ranges between $90^{\circ}$ and $180^{\circ}$.

The control loop of SVC uses an auxiliary stabilizing signal. To improve system stability, a speed deviation signal was chosen as an input signal. The structure of the SVC auxiliary controller is similar to PSS and composed of the two lead-lag blocks, a washout block and a gain block.

As illustrated in Figure 10, the structure of the proposed SVC-based POD with a leadlag compensator consists of a gain block $K_{S V C}$, a signal washout block, and two-stage phasecompensation blocks. The dynamic equation of the effective susceptance of the SVC can be expressed by Equation (18). $\mathrm{Kr}$ and $\mathrm{Tr}$ are, respectively, the gain $K_{r}$ and $T_{r}$ time constant of 
the thyristors firing control system. To improve the electromechanical oscillations, the input signal of the POD stabilizer is relatively selected to the speed deviation of the machines.

$$
\dot{B}_{e}=\frac{1}{T_{r}}\left(-B_{e}+K_{r}\left(V_{r e f}-V_{t}+V_{s}\right)\right)
$$

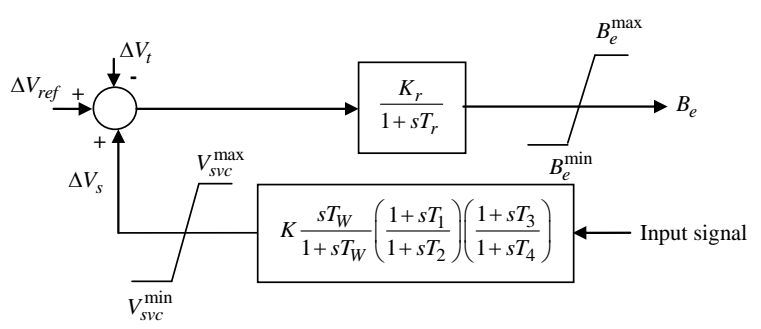

Figure 10. Block diagram of SVC.

\subsubsection{Damping Controllers Design}

In this study, the purpose is to tune simultaneously the parameters of the PSS and SVC controllers that provide the optimum system stability. The controller's parameters are obtained for the minimum Integral of Time multiply Absolute Error (ITAE) performance index. The previous performance criterion is stated as follows:

$$
J=\sum_{j=1}^{N G} \sum_{i=1}^{N P} \int_{0}^{t_{s i m}} t\left|\Delta \omega_{i}\right| \mathrm{d} t .
$$

where $t_{\text {sim }}$ is the simulation time, $\Delta \omega$ is the speed deviation of the machi, $N_{P}$ and $N_{G}$ are the operating points and the generators numbers, respectively. The optimization problem consisting of parameters of controllers design is stated as follows:

Minimize $J$ subject to

$$
\begin{aligned}
& K^{\text {max }} \leq K \leq K^{\text {min }} \\
& T_{1 i}^{\text {min }} \leq T_{1 i} \leq T_{1 i}^{\text {max }} \\
& T_{2 i}^{\text {min }} \leq T_{2 i} \leq T_{2 i}^{\text {max }} \\
& T_{3 i}^{\text {min }} \leq T_{3 i} \leq T_{3 i}^{\text {max }} \\
& T_{4 i}^{\text {min }} \leq T_{4 i} \leq T_{4 i}^{\text {max }}
\end{aligned}
$$

The value of the washout time constant is ordinarily predetermined $T_{\omega}=5 \mathrm{~s}$ to reduce the required time to achieve the final solution. According to the previous works, the gain of the controller's range is [1-100]. The values of the lead-lag time constants range are [0.05-1.5]. Generally, significant computational time is gained when $T_{2}$ and $T_{4}$ are predetermined. In several power system stability design works, the time constants $T_{2}=T_{4}=0.05[54-56]$.

Three operating conditions are considered for the WSCC test system in the design process (see Table 11).

The PSSs and SVC parameters for the three operating conditions are optimized by the HTLBO technique. The design approach was applied for the coordinated design, PSSs only and SVC only. The optimum controllers parameters are given in Table 12). 
Table 11. Operating conditions for the power system under study.

\begin{tabular}{lllllll}
\hline Generator & \multicolumn{2}{l}{ Nom.Loading } & \multicolumn{2}{l}{ Heavy Loading } & \multicolumn{2}{l}{ Light Loading } \\
\hline & $\mathbf{P}(\mathbf{p u})$ & $\mathbf{Q}(\mathbf{p u})$ & $\mathbf{P}(\mathbf{p u})$ & $\mathbf{Q}(\mathbf{p u})$ & $\mathbf{P}(\mathbf{p u})$ & $\mathbf{Q}(\mathbf{p u})$ \\
\hline Gene. 1 & 0.72 & 0.27 & 2.21 & 1.09 & 0.36 & 0.16 \\
Gene. 2 & 1.63 & 0.07 & 1.92 & 0.56 & 0.80 & -0.11 \\
Gene. 3 & 0.85 & -0.11 & 1.28 & 0.36 & 0.45 & -0.20 \\
Load & & & & & & \\
Load A & 1.25 & 0.50 & 2.00 & 0.80 & 0.65 & 0.55 \\
Load B & 0.90 & 0.30 & 1.80 & 0.60 & 0.45 & 0.35 \\
Load C & 1.00 & 0.35 & 1.50 & 0.60 & 0.50 & 0.25 \\
\hline
\end{tabular}

Table 12. Controllers' optimal parameters tuned by HTLBO.

\begin{tabular}{lllllll}
\hline & \multicolumn{3}{l}{ Uncoordinated Design } & \multicolumn{3}{l}{ Coordinated Design } \\
\hline & PSS1 & PSS2 & SVC & PSS1 & PSS2 & SVC \\
\hline K & 14.0006 & 7.3196 & 120.4268 & 24.011 & 17.3196 & 22.5241 \\
T1 & 0.3282 & 0.1945 & 0.2013 & 0.3270 & 0.1922 & 0.7840 \\
T2 & 0.05 & 0.05 & 0.05 & 0.0500 & 0.0500 & 0.0500 \\
T3 & 0.0754 & 0.5846 & 0.3687 & 0.0766 & 0.5776 & 1.2527 \\
T4 & 0.05 & 0.05 & 0.05 & 0.0500 & 0.0500 & 0.0500 \\
\hline
\end{tabular}

Figure 11 depicted the variations of the objective function with the standard and the hyperchaotic TLBO techniques. The HTLBO gives faster convergence than TLBO, which demonstrates the potential of the proposed design approach.

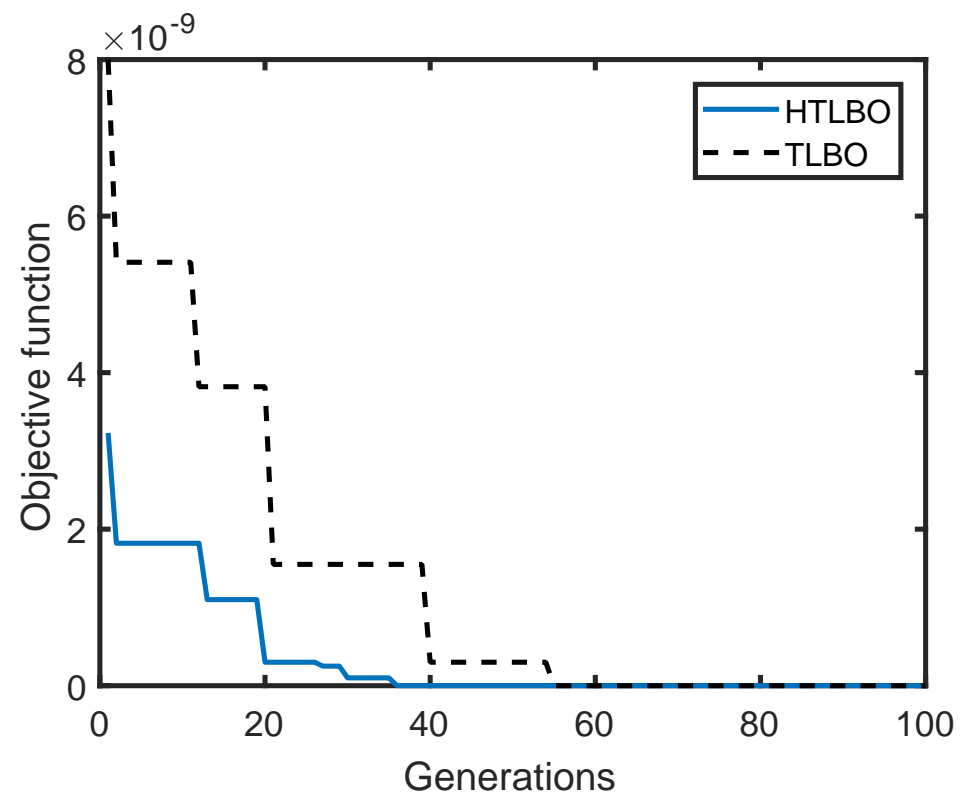

Figure 11. Variations of objective function for TLBO and HTLBO algorithms.

\subsection{Simulation Results}

To assess the robustness of the HTLBO technique, the controller is tested under severe conditions and different operating conditions. The coordinated tuning of controllers is compared with PSSs and SVC tuned individually. In this paper, power system numerical simulations have been carried out using the fourth-order Runge-Kutta method with the software Matlab. Time-domain simulation is performed for the following three scenarios: 


\section{Scenario1 :}

The nonlinear simulations are performed for different loading conditions under a $10 \%$ increase in mechanical torque at $t=1 \mathrm{~s}$. The speed deviation of the synchronous machines is shown in Figures 12 and 13. The system's response with the coordinate PSSs and SVC controllers is damped compared with HTLBOPSSs only and HTLBOSVC only.

\section{Scenario2 :}

A severe disturbance consists of a three-phase fault located at the end of transmission line $5 \# 7$ during $100 \mathrm{~ms}$. The original WSCC test system is restored when the fault is cleared. The system response is shown in Figures 14 and 15 revealed that the coordination design of HTLBOPSSs and HTLBOSVC controllers outperform the former stabilizers tuned individually.
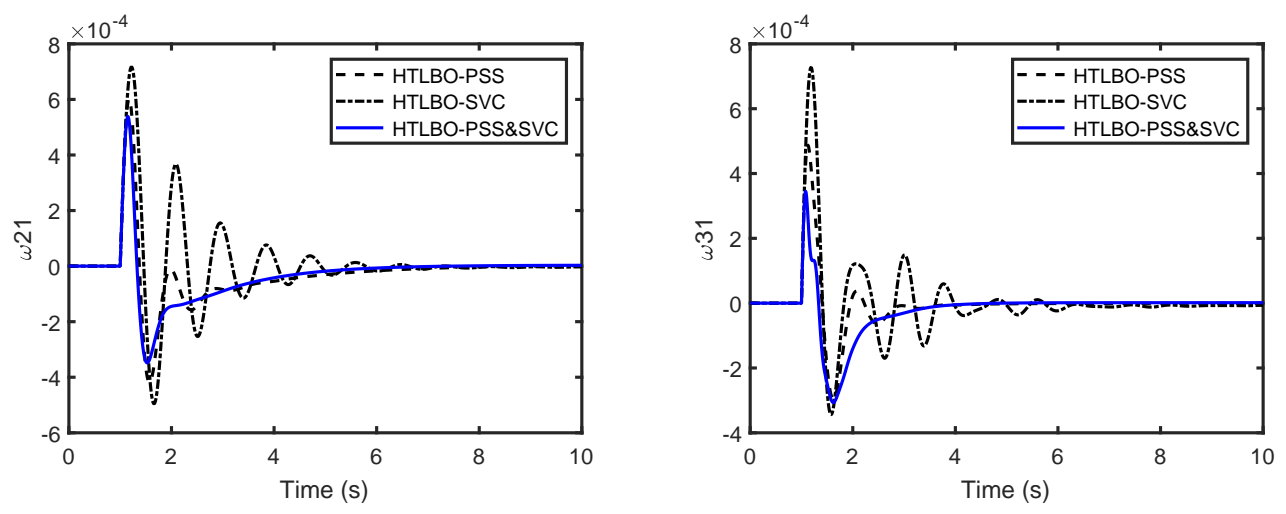

Figure 12. Response of $\triangle \omega 21$ and $\triangle \omega 31$ for light load condition under scenario1.
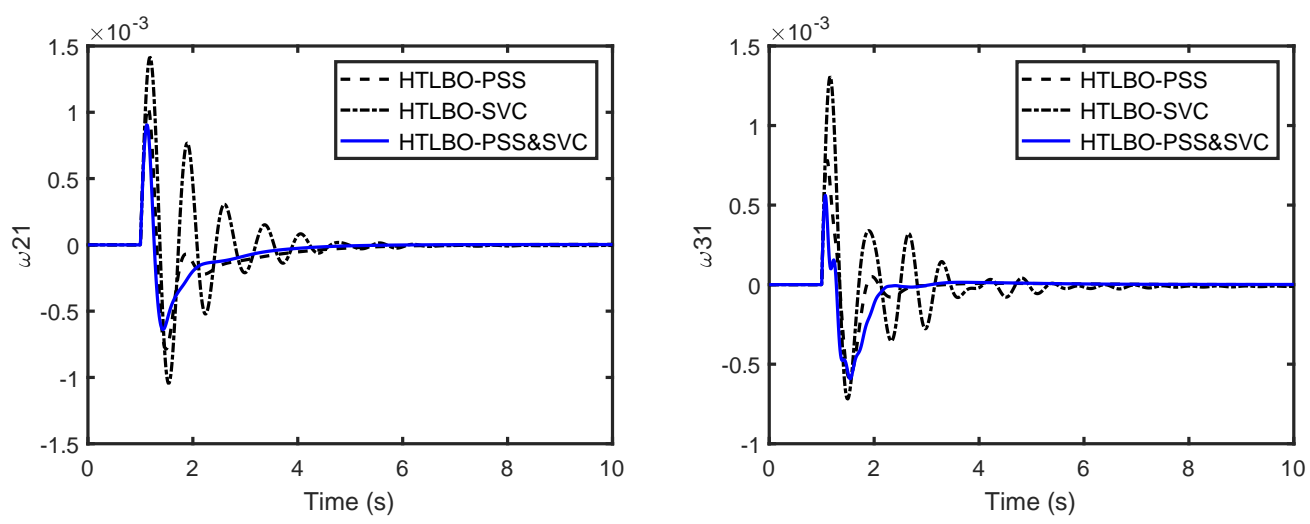

Figure 13. Response of $\triangle \omega 21$ and $\triangle \omega 31$ for nominal load condition under scenario1.
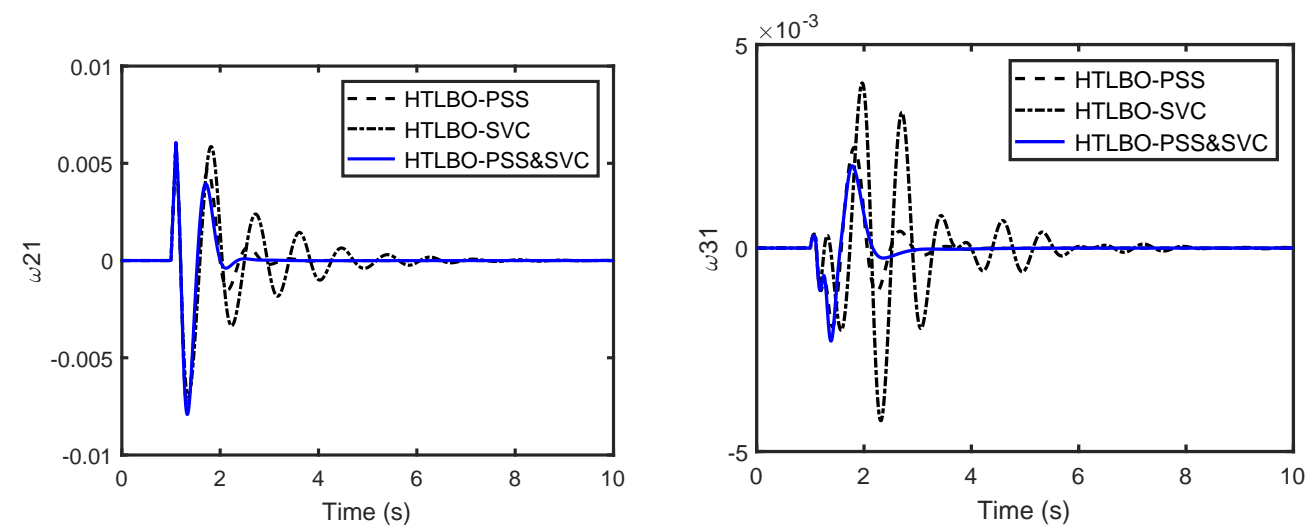

Figure 14. Response of $\triangle \omega 21$ and $\triangle \omega 31$ for light load condition under scenario2. 

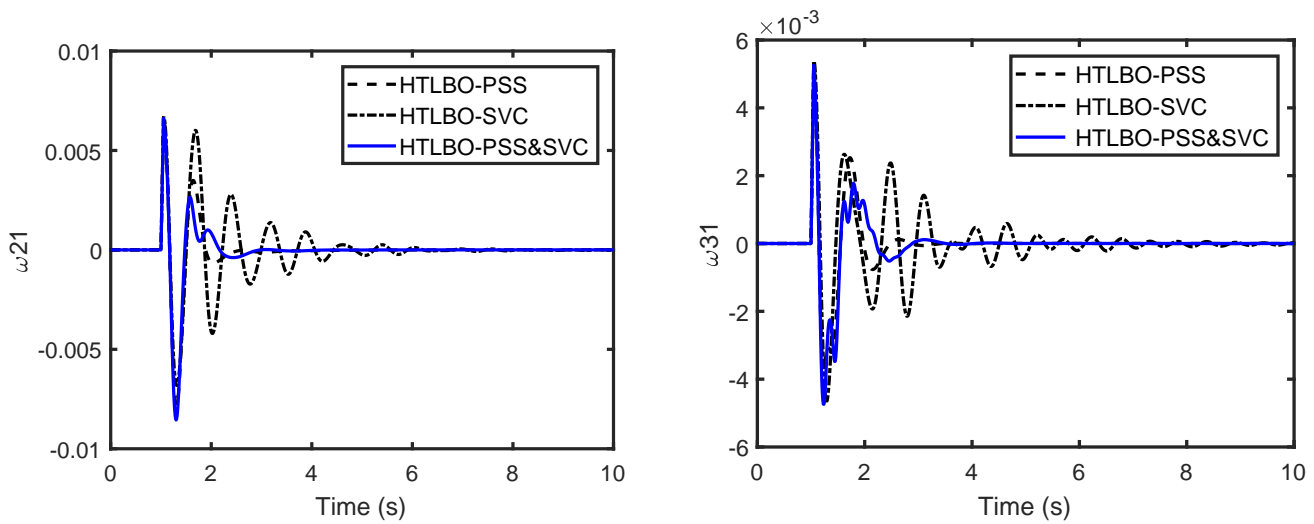

Figure 15. Response of $\triangle \omega 21$ and $\triangle \omega 31$ for heavy load condition under scenario2.

\section{Scenario3:}

The test system is under a six-cycle three-phase fault. The initial configuration of the system is not restored since the line fault $5 \# 7$ is tripped permanently. Our approach is evaluated by applying the HTLBO in the tuning of the stabilizers individually and simultaneously. Figures 16 and 17 demonstrate the ability of the stabilizers to provide a satisfactory damping of electromechanical oscillations under a harsh condition.
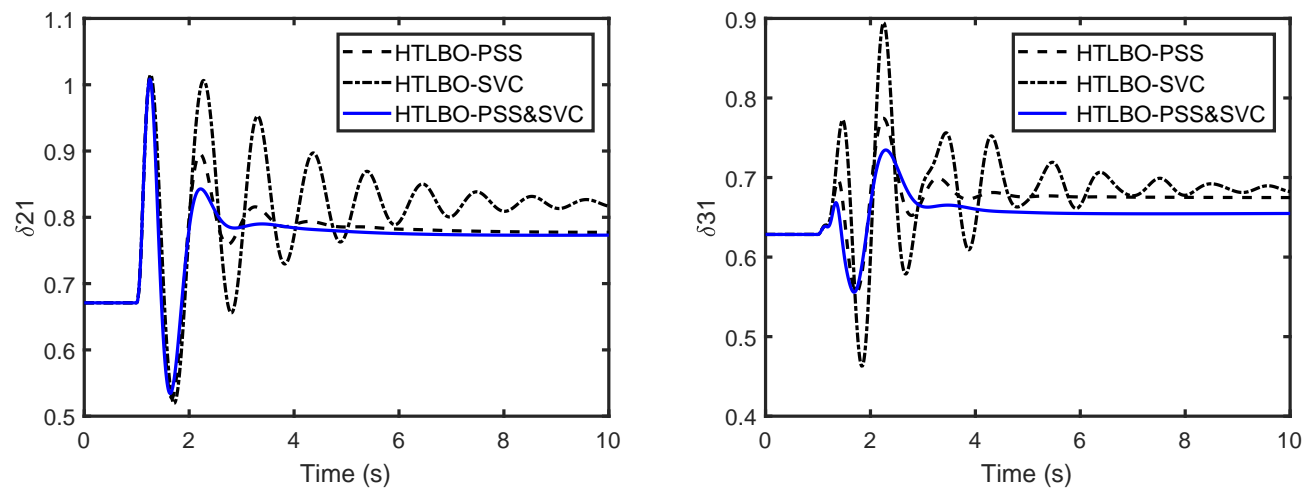

Figure 16. Response of $\triangle \delta 21$ and $\triangle \delta 31$ for light load condition under scenario3.
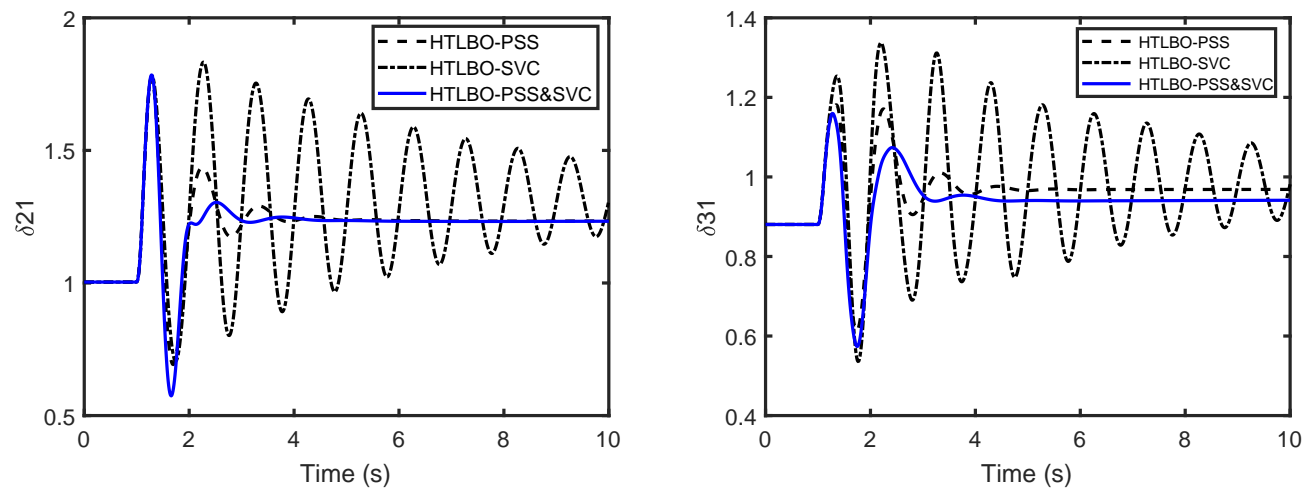

Figure 17. Response of $\triangle \delta 21$ and $\triangle \delta 31$ for nominal load condition under scenario3.

The Integral of Absolute Error (IAE) is involved in our study to quantify the stabilizers' quality. The mathematical expression of the previous criterion is:

$$
J=\int_{0}^{t} \sum_{i=1}^{N G}\left|\Delta \omega_{i}\right| \mathrm{d} t
$$


It is worth mentioning that better time-domain behavior is characterized by the lowest values of IAE. Table 13 shows the values of the IAE criterion for all considered scenarios and operating systems. The smaller values of IAE obtained by HTLBOPSS\&SVC controllers reflect its superiority in damping the rotor oscillations.

Table 13. IAE values for the suggested scenarios.

\begin{tabular}{lllll}
\hline Fault & Method & Normal Load & Heavy Load & Light Load \\
\hline Scenario1 & HTLBOPSS & 0.01499 & 0.01616 & 0.00685 \\
& HTBOSVC & 0.03068 & 0.05084 & 0.01379 \\
Scenario2 & ITLAPSS\&SVC & $\mathbf{0 . 0 1 2 2 8}$ & $\mathbf{0 . 0 1 2 7 5}$ & $\mathbf{0 . 0 0 5 0 1}$ \\
& HTLBOPSS & 0.01683 & 0.01699 & 0.00744 \\
& HTLBOSVC & 0.12016 & 0.23058 & 0.04210 \\
Scenario3 & HTLBOPSS\&SVC & $\mathbf{0 . 0 1 4 8 7}$ & $\mathbf{0 . 0 2 3 0 6}$ & $\mathbf{0 . 0 0 5 4 3}$ \\
& HTLBOPSS & 0.00115 & 0.00116 & 0.00079 \\
& HTLBOSVC & 0.09221 & 0.18403 & 0.03103 \\
& HTLBOPSS\&SVC & $\mathbf{0 . 0 0 1 0 4}$ & $\mathbf{0 . 0 0 1 1 0}$ & $\mathbf{0 . 0 0 0 7 8}$ \\
\hline
\end{tabular}

Table 14 exhibits the electromechanical mode and the damping ratio of the WSCC test system for the above operating conditions. The smallest damping ratio with HTLBOSVC modes for normal, heavy and light are: $(\xi=0.0536, \xi=0.0416, \xi=0.0783)$. The HTLBOPSSs provide a satisfactory damping performance compared to HTLBOSVC. Moreover, the coordinated PSSs and SVC designed with the proposed HTLBO enhance significantly the damping ratio to be $(\xi=0.4586, \xi=0.5760, \xi=0.6302)$ for the aforementioned conditions. It can therefore be concluded that our approach provides a satisfactory damping performance since the modes of the system are substantially shifted to the left of the s-plane.

A comparative of system eigenvalues and damping ratio of mechanical modes with light, normal, and heavy loading for the proposed HTLBO and Teaching-Learning (TL) algorithm given in [57] is shown in Table 14. The damping ratios corresponding to the HTLBO controller are greater than that corresponding to the TL algorithm. The eigenvalue analysis demonstrates that the proposed controller provides a satisfactory damping performance.

Table 14. Electromechanical modes and damping ratios under different loading Conditions and stabilizers.

\begin{tabular}{cccc}
\hline & Normal Load & Light Load & Heavy Load \\
\hline HTLBOPSSs & $-2.5227 \pm 8.4257 i, 0.2868$ & $-4.1636 \pm 6.8816 i ; 0.5177$ & $-5.4270 \pm 7.9166 i ; 0.5654$ \\
& $-4.8766 \pm 7.5776 i, 0.5412$ & $-1.9237 \pm 7.3739 i ; 0.2524$ & $-1.7399 \pm 7.9454 i ; 0.2139$ \\
& & & $-0.4976 \pm 11.9566 i ; 0.0416$ \\
HTLBOSVC & $-0.6322 \pm 11.7889 i ; 0.0536$ & $-0.7595 \pm 9.6653 i ; 0.0783$ & $-0.5417 \pm 7.6075 i ; 0.0710$ \\
& $-0.7981 \pm 8.0646 i ; 0.0985$ & $-0.6840 \pm 6.8761 ; ; 0.0990$ & $-4.3362 \pm 9.9705 i ; 0.3988$ \\
TLPSS\&SVC [57] & $-3.8461 \pm 11.6327 i ; 0.3139$ & $-4.3571 \pm 12.5613 i ; 0.3277$ & $-6.2342 \pm 5.0439 i ; 0.7774$ \\
& $-4.8813 \pm 2.7527 i ; 0.8710$ & $-3.5977 \pm 04.5640 i ; 0.6191$ & $-3.8856 \pm 2.7691 i ; 0.8144$ \\
HTLBOPSS\&SVC & & & $-4.2187 \pm 7.3542 i ; 0.4976$ \\
\hline
\end{tabular}

\section{Conclusions}

This work presents a new technique called the Hyperchaotic Teaching-Learning-Based Optimization algorithm known as HTLBO. The proposed algorithm improves the overall performance of the original counterpart by involving three approaches, i.e., replacement of the random numbers in search equations by the hyperchaotic sequences generated by the hyperchaotic map, implementation of three new mutation equations, and hyperchaotic perturbation. The latter favors the global search, whereas the mutation strategies guide 
the algorithm towards promising regions and guarantee an intensification effect at the course-end of the algorithm. That is, the mutation strategies favor local search. Therefore, a balance between diversification and intensification effects is provided during the algorithm. The proposed algorithm is tested on twenty-six test functions. The obtained results confirm that HTLBO provides competitive results compared to four well-known optimization algorithms. The exploitation and exploration capabilities of the HTBO algorithm are evaluated using multimodal, unimodal, and rotated test problems. Indeed, the unimodal functions have only one local optimum; thus, they are suitable for evaluating the exploitation capability. Whereas the multimodal functions present many local optima, they are ideal for investigating the exploration characteristic. The rotated test functions demonstrate the ability of HTLBO to solve complex problems. Furthermore, the proposed algorithm is employed to solve the coordination design of PSSs and SVC for a multimachine power system. The robustness is assessed via three operating conditions. The nonlinear simulations and the eigenvalue analysis confirm that the proposed approach provided an excellent damping behavior than the uncoordinated scheme.

Author Contributions: Conceptualization, A.F. and A.B.; methodology, K.A. and B.M.A.; software, A.B. and A.F.; validation, A.F., A.B. and A.A.; formal analysis, R.A., M.B.B.H. and B.M.A.; investigation, K.A. and R.A.; writing - original draft preparation, A.F., B.M.A. and A.B.; supervision, K.A. and A.A.; project administration, B.M.A.; funding acquisition, A.F. All authors have read and agreed to the published version of the manuscript.

Funding: This research has been funded by Scientific Research Deanship at University of Ha'ilSaudi Arabia through project number RG-20 106.

Institutional Review Board Statement: Not applicable.

Informed Consent Statement: Not applicable.

Data Availability Statement: Not applicable.

Conflicts of Interest: The authors declare no conflict of interest.

$\begin{array}{ll}\text { Abbreviations } & \\ T_{F} & \text { Teacher factor } \\ x_{d i}^{\prime} & \text { d-axis transient reactance } \\ r & \text { Random number } \\ x_{q i} & \text { Roxis reactance of the } i \text { th machine } \\ \delta_{i} & \text { q-axis armature current of machine } \\ i_{q i} & \text { Rotor speed of the } i \text { th synchronous machine } \\ \omega_{i} & \text { gain of the regulator } \\ K_{A} & \text { Base frequency in rad /sec } \\ \omega_{b} & \text { time constant of the regulator } \\ T_{A} & \text { Internal voltage of the } i \text { th machine } \\ E_{q i}^{\prime} & \text { voltage reference } \\ V_{r e f} & \text { Field voltage of the } i \text { th machine } \\ E_{f d} & \text { terminal voltage } \\ V_{t} & \text { Inertia constant of the } i \text { th machine } \\ M_{i} & \text { time constants of controller } \\ T_{1}-T_{4} & \text { Damping coefficient of the } i \text { th machine } \\ D_{i} & \text { SVC fixed inductor reactance } \\ X_{L} & \text { Time constant of } i \text { th machine excitation circuit } \\ T_{d 0 i}^{\prime} & \text { firing angle of the thyristor } \\ \alpha & \text { Mechanical torque of the } i \text { th machine } \\ T_{m i} & \text { Hyperchaotic sequence } \\ H c j & \end{array}$




$\begin{array}{ll}T_{e i} & \text { Electric torque of the } i \text { th machine } \\ x_{d} & \text { d-axis reactance } \\ X_{e} & \text { Effective reactance of the SVC } \\ K_{S V C} & \text { Gain of the SVC } \\ K_{r} & \text { Gain of the thyristors firing control system } \\ T_{r} & \text { Time constant of the thyristors firing control system } \\ N P & \text { Number of operating points } \\ N G & \text { Generators numbers } \\ t_{s i m} & \text { Simulation time } \\ T_{\omega} & \text { time constant of the washout block }\end{array}$

\section{References}

1. Naderi, E.; Pourakbari-Kasmaei, M.; Abdi, H. An efficient particle swarm optimization algorithm to solve optimal power flow problem integrated with FACTS devices. Appl. Soft Comput. 2019, 80, 243-262. [CrossRef]

2. Farah, A.; Guesmi, T.; Abdallah, H.H.; Ouali, A. A novel chaotic teaching-Learning-based optimization algorithm for multimachine power system stabilizers design problem. Int. J. Electr. Power Energy Syst. 2016, 77, 197-209. [CrossRef]

3. Dehghani, M.; Mardaneh, M.; Guerrero, J.M.; Malik, O.P.; Ramirez-Mendoza, R.A.; Matas, J.; Vasquez, J.C.; Parra-Arroyo, L. A New "Doctor and Patient" Optimization Algorithm: An Application to Energy Commitment Problem. Appl. Sci. 2020, 10, 5791. [CrossRef]

4. Bahmani-Firouzi, B.; Azizipanah-Abarghooee, R. Optimal sizing of battery energy storage for micro-grid operation management using a new improved bat algorithm. Int. J. Electr. Power Energy Syst. 2014, 56, 42-54. [CrossRef]

5. Yildiz, A.R.; Kaya, N.; Ozturk, F.; Alankus, O. Optimal design of vehicle components using topology design and optimisation. Int. J. Veh. Des. 2004, 34, 387-398. [CrossRef]

6. Kundur, P.; Balu, N.J.; Lauby, M.G. Power System Stability and Control; McGraw-Hill: New York, NY, USA, $1994 ;$ Volume 7.

7. Ghasemi, A.; Shayeghi, H.; Alkhatib, H. Robust design of multimachine power system stabilizers using fuzzy gravitational search algorithm. Int. J. Electr. Power Energy Syst. 2013, 51, 190-200. [CrossRef]

8. Hingorani, N.G. FACTS-flexible AC transmission system. In Proceedings of the International Conference on AC and DC Power Transmission, London, UK, 17-20 September 1991; IET: London, UK, 1991; pp. 1-7.

9. Anderson, P.M.; Fouad, A.A. Power System Control and Stability; John Wiley \& Sons: Hoboken, NJ, USA, 2008.

10. Peres, W.; Júnior, I.C.S.; Passos Filho, J.A. Gradient based hybrid metaheuristics for robust tuning of power system stabilizers. Int. J. Electr. Power Energy Syst. 2018, 95, 47-72. [CrossRef]

11. Goldberg, D.E.; Holland, J.H. Genetic algorithms and machine learning. Mach. Learn. 1988, 3, 95-99. [CrossRef]

12. Noktehdan, A.; Karimi, B.; Kashan, A.H. A differential evolution algorithm for the manufacturing cell formation problem using group based operators. Expert Syst. Appl. 2010, 37, 4822-4829. [CrossRef]

13. Espejo, P.G.; Ventura, S.; Herrera, F. A survey on the application of genetic programming to classification. IEEE Trans. Syst. Man Cybern. Part C Appl. Rev. 2009, 40, 121-144. [CrossRef]

14. Bhattacharya, A.; Chattopadhyay, P.K. Biogeography-based optimization for different economic load dispatch problems. IEEE Trans. Power Syst. 2009, 25, 1064-1077. [CrossRef]

15. Eberhart, R.; Kennedy, J. Particle swarm optimization. In Proceedings of the IEEE International Conference on Neural Networks, Perth, WA, Australia, 27 November-1 December 1995; Volume 4, pp. 1942-1948.

16. Stützle, T. Ant colony optimization. In Proceedings of the International Conference on Evolutionary Multi-Criterion Optimization, Nantes, France, 7-10 April 2009; Springer: Berlin/Heidelberg, Germany, 2009; p. 2.

17. Yang, X.S.; Deb, S. Cuckoo search via Lévy flights. In Proceedings of the 2009 World Congress on Nature \& Biologically Inspired Computing (NaBIC), Coimbatore, India, 9-11 December 2009; IEEE: New York, NY, USA, 2009; pp. $210-214$.

18. Karaboga, D.; Basturk, B. A powerful and efficient algorithm for numerical function optimization: Artificial bee colony (ABC) algorithm. J. Glob. Optim. 2007, 39, 459-471. [CrossRef]

19. Yang, X.S. Firefly algorithms for multimodal optimization. In International Symposium on Stochastic Algorithms; Springer: Berlin/Heidelberg, Germany, 2009; pp. 169-178.

20. Yang, X.S. A new metaheuristic bat-inspired algorithm. In Nature Inspired Cooperative Strategies for Optimization (NICSO 2010); Springer: Berlin/Heidelberg, Germany, 2010; pp. 65-74.

21. Geem, Z.W.; Kim, J.H.; Loganathan, G.V. A new heuristic optimization algorithm: Harmony search. Simulation 2001, 76, 60-68. [CrossRef]

22. Kaveh, A.; Talatahari, S. Optimum design of skeletal structures using imperialist competitive algorithm. Comput. Struct. 2010, 88, 1220-1229. [CrossRef]

23. Tan, Y.; Zhu, Y. Fireworks algorithm for optimization. In Proceedings of the International Conference in Swarm Intelligence, Beijing, China, 12-15 June 2010; Springer: Berlin/Heidelberg, Germany, 2010; pp. 355-364.

24. Dai, C.; Zhu, Y.; Chen, W. Seeker optimization algorithm. In Proceedings of the International Conference on Computational and Information Science, Guangzhou, China, 3-6 November 2006; Springer: Berlin/Heidelberg, Germany, 2006; pp. 167-176. 
25. Gandomi, A.H. Interior search algorithm (ISA): A novel approach for global optimization. ISA Trans. 2014, 53, 1168-1183. [CrossRef]

26. Rao, R.V.; Savsani, V.J.; Vakharia, D. Teaching_Learning-based optimization: A novel method for constrained mechanical design optimization problems. Comput.-Aided Des. 2011, 43, 303-315. [CrossRef]

27. García, J.A.M.; Mena, A.J.G. Optimal distributed generation location and size using a modified teaching-learning based optimization algorithm. Int. J. Electr. Power Energy Syst. 2013, 50, 65-75. [CrossRef]

28. Ghasemi, M.; Ghavidel, S.; Gitizadeh, M.; Akbari, E. An improved teaching—Learning-based optimization algorithm using Lévy mutation strategy for non-smooth optimal power flow. Int. J. Electr. Power Energy Syst. 2015, 65, 375-384. [CrossRef]

29. Rao, R.; Patel, V. An elitist teaching-learning-based optimization algorithm for solving complex constrained optimization problems. Int. J. Ind. Eng. Comput. 2012, 3, 535-560. [CrossRef]

30. Kadambur, R.; Kotecha, P. Multi-level production planning in a petrochemical industry using elitist Teaching-Learning-BasedOptimization. Expert Syst. Appl. 2015, 42, 628-641. [CrossRef]

31. Satapathy, S.C.; Naik, A. Modified Teaching-Learning-Based Optimization algorithm for global numerical optimization-A comparative study. Swarm Evol. Comput. 2014, 16, 28-37. [CrossRef]

32. Chen, C.H. Group leader dominated teaching-learning based optimization. In Proceedings of the 2013 International Conference on Parallel and Distributed Computing, Applications and Technologies, Taipei, Taiwan, 16-18 December 2013; IEEE: New York, NY, USA, 2013; pp. 304-308.

33. Yang, Z.; Li, K.; Foley, A.; Zhang, C. A New Self-Learning TLBO Algorithm for RBF Neural Modelling of Batteries in Electric Vehicles. In Proceedings of the 2014 IEEE Congress on Evolutionary Computation (CEC), Beijing, China, 6-11 July 2014 ; IEEE: New York, NY, USA, 2014; pp. 2685-2691.

34. Uzlu, E.; Kömürcü, M.İ; Kankal, M.; Dede, T.; Öztürk, H.T. Prediction of berm geometry using a set of laboratory tests combined with teaching-Learning-based optimization and artificial bee colony algorithms. Appl. Ocean. Res. 2014, 48, 103-113. [CrossRef]

35. Ghasemi, M.; Ghanbarian, M.M.; Ghavidel, S.; Rahmani, S.; Moghaddam, E.M. Modified teaching learning algorithm and double differential evolution algorithm for optimal reactive power dispatch problem: A comparative study. Inf. Sci. 2014, 278, 231-249. [CrossRef]

36. Eslami, M.; Shareef, H.; Mohamed, A.; Khajehzadeh, M. Coordinated design of PSS and SVC damping controller using CPSO. In Proceedings of the 2011 5th International Power Engineering and Optimization Conference, Shah Alam, Malaysia, 6-7 June 2011; IEEE: New York, NY, USA, 2011; pp. 11-16.

37. Farahani, M. Intelligent control of SVC using wavelet neural network to enhance transient stability. Eng. Appl. Artif. Intell. 2013, 26, 273-280. [CrossRef]

38. Farsangi, M.M.; Nezamabadi-pour, H.; Song, Y.H.; Lee, K.Y. Placement of SVCs and selection of stabilizing signals in power systems. IEEE Trans. Power Syst. 2007, 22, 1061-1071. [CrossRef]

39. Yang, C.F.; Lai, G.G.; Lee, C.H.; Su, C.T.; Chang, G.W. Optimal setting of reactive compensation devices with an improved voltage stability index for voltage stability enhancement. Int. J. Electr. Power Energy Syst. 2012, 37, 50-57. [CrossRef]

40. Ali, E.; Abd-Elazim, S.M. Power system stability enhancement via new coordinated design of PSSs and SVC. Int. J. WSEAS Trans. Power Syst. 2014, 9, 428-438.

41. Darabian, M.; Mohseni-Bonab, S.M.; Mohammadi-Ivatloo, B. Improvement of power system stability by optimal SVC controller design using shuffled frog-leaping algorithm. IETE J. Res. 2015, 61, 160-169. [CrossRef]

42. Gitizadeh, M.; Ghavidel, S. Improving transient stability with multi-objective allocation and parameter setting of SVC in a multi-machine power system. IETE J. Res. 2014, 60, 33-41. [CrossRef]

43. Abd-Elazim, S.M.; Ali, E.S. Power system stability enhancement via bacteria foraging optimization algorithm. Arab. J. Sci. Eng. 2013, 38, 599-611. [CrossRef]

44. Alatas, B. Chaotic bee colony algorithms for global numerical optimization. Expert Syst. Appl. 2010, 37, 5682-5687. [CrossRef]

45. Rizk-Allah, R.M.; Hassanien, A.E.; Bhattacharyya, S. Chaotic crow search algorithm for fractional optimization problems. Appl. Soft Comput. 2018, 71, 1161-1175. [CrossRef]

46. Liu, B.; Wang, L.; Jin, Y.H.; Tang, F.; Huang, D.X. Improved particle swarm optimization combined with chaos. Chaos Solitons Fractals 2005, 25, 1261-1271. [CrossRef]

47. Farah, A.; Belazi, A. A novel chaotic Jaya algorithm for unconstrained numerical optimization. Nonlinear Dyn. 2018, 93, 1451-1480. [CrossRef]

48. Chakraborty, U.K. Semi-Steady-State Jaya Algorithm for Optimization. Appl. Sci. 2020, 10, 5388. [CrossRef]

49. Migallón, H.; Jimeno-Morenilla, A.; Sánchez-Romero, J.L.; Belazi, A. Efficient parallel and fast convergence chaotic Jaya algorithms. Swarm Evol. Comput. 2020, 56, 100698. [CrossRef]

50. Migallón, H.; Jimeno-Morenilla, A.; Rico, H.; Sánchez-Romero, J.; Belazi, A. Multi-level parallel chaotic Jaya optimization algorithms for solving constrained engineering design problems. J. Supercomput. 2021, 77, 12280-12319. [CrossRef]

51. Gao, S.; Vairappan, C.; Wang, Y.; Cao, Q.; Tang, Z. Gravitational search algorithm combined with chaos for unconstrained numerical optimization. Appl. Math. Comput. 2014, 231, 48-62. [CrossRef]

52. Rao, R.V.; Savsani, V.J.; Vakharia, D. Teaching-Learning-based optimization: An optimization method for continuous non-linear large scale problems. Inf. Sci. 2012, 183, 1-15. [CrossRef] 
53. Yang, Q.; Bai, M. A new 5D hyperchaotic system based on modified generalized Lorenz system. Nonlinear Dyn. 2017, 88, 189-221. [CrossRef]

54. Ali, E.; Abd-Elazim, S. Coordinated design of PSSs and TCSC via bacterial swarm optimization algorithm in a multimachine power system. Int. J. Electr. Power Energy Syst. 2012, 36, 84-92. [CrossRef]

55. Abd-Elazim, S.; Ali, E. Bacteria foraging optimization algorithm based SVC damping controller design for power system stability enhancement. Int. J. Electr. Power Energy Syst. 2012, 43, 933-940. [CrossRef]

56. Panda, S.; Yegireddy, N.K.; Mohapatra, S.K. Hybrid BFOA-PSO approach for coordinated design of PSS and SSSC-based controller considering time delays. Int. J. Electr. Power Energy Syst. 2013, 49, 221-233. [CrossRef]

57. Welhazi, Y.; Guesmi, T.; Abdallah, H.H. Coordinated Tuning of SVC and PSSs in Power System using Teaching Learning Based Algorithm. In Proceedings of the 10th International Renewable Energy Congress (IREC), Sousse, Tunisia, 26-28 March 2019; IEEE: New York, NY, USA, 2019; pp. 1-6. 